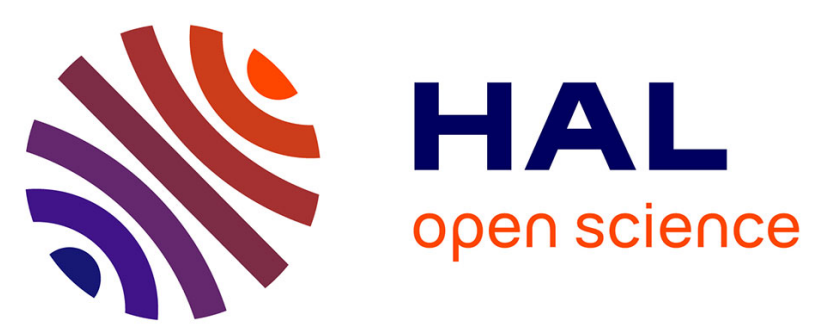

\title{
The posterior parietal cortex processes visuo-spatial and extra-retinal information for saccadic remapping: A case study
}

Alexis Cheviet, Laure Pisella, Denis Pélisson

\section{- To cite this version:}

Alexis Cheviet, Laure Pisella, Denis Pélisson. The posterior parietal cortex processes visuo-spatial and extra-retinal information for saccadic remapping: A case study. Cortex, 2021, 139, pp.134-151. 10.1016/j.cortex.2021.02.026 . hal-03373951

\section{HAL Id: hal-03373951 \\ https://hal.science/hal-03373951}

Submitted on 20 Oct 2021

HAL is a multi-disciplinary open access archive for the deposit and dissemination of scientific research documents, whether they are published or not. The documents may come from teaching and research institutions in France or abroad, or from public or private research centers.
L'archive ouverte pluridisciplinaire HAL, est destinée au dépôt et à la diffusion de documents scientifiques de niveau recherche, publiés ou non, émanant des établissements d'enseignement et de recherche français ou étrangers, des laboratoires publics ou privés. 


\title{
The posterior parietal cortex processes visuo-spatial and extra-retinal information for saccadic remapping: a case study
}

\author{
Alexis Cheviet ${ }^{\mathrm{a}}$ \\ a : IMPACT Team, Lyon Neuroscience Research Center, INSERM, U1028; CNRS, \\ UMR5292; University of Lyon, F-69000. \\ 16, av. du Doyen Lépine, 69676 Bron cedex, France
}

\section{Laure Pisella ${ }^{\mathbf{b}_{*}}$}

b : IMPACT Team, Lyon Neuroscience Research Center, INSERM, U1028; CNRS, UMR5292; University of Lyon, F-69000.

16, av. du Doyen Lépine, 69676 Bron cedex, France

laure.pisella@inserm.fr

\author{
and Denis Pélisson ${ }^{\mathfrak{c}_{*}}$ \\ c : IMPACT Team, Lyon Neuroscience Research Center, INSERM, U1028; CNRS, \\ UMR5292; University of Lyon, F-69000. \\ 16, av. du Doyen Lépine, 69676 Bron cedex, France \\ denis.pelisson@inserm.fr
}

*co-last authors

\section{Correspondance:}

Alexis Cheviet: alexis.cheviet@inserm.fr

Lyon Neuroscience Research Center, IMPACT Team, Bât INSERM, 16, av. du Doyen Lépine, 69676 Bron cedex, France 


\section{Highlights}

- Lesion of the right PPC led to an improper localization of visual stimuli in the contralateral visual hemifield

- Deficits were characterized first by an improper computation of the to-be-localized stimulus position under gaze fixation

- Additional transsaccadic deficits emerged when the task further required extra-retinal saccadic signals

- Trans-saccadic deficits are consistent with an underestimation of saccade size predicted from extra-retinal signals 


\begin{abstract}
Optimally collecting information and controlling behaviour require that we constantly scan our visual environment through eye movements. How the dynamic interaction between shortlived retinal images and extra-retinal signals of eye motion results in our subjective experience of visual stability remains a major issue in Cognitive Neuroscience. The present study aimed to assess and determine the nature of the contribution of the posterior parietal cortex (PPC) to the saccadic remapping mechanisms which contribute to such perceptual visual constancy. Perceptual responses in transsaccadic visual localization tasks were measured in a patient presenting with a PPC lesion and manifesting optic ataxia in the left hemifield with no neglect. Two perceptual localization tasks, each with versus without an intervening saccade, were used: the saccadic suppression of displacement (SSD) task (Ostendorf, Liebermann \& Ploner, 2010) and the peri-saccadic flash localization (LOC) task (Zimmermann \& Lappe, 2010). Compared to a group of age-matched healthy subjects, the patient showed a specific pattern of perceptual deficits in the ataxic (left) hemifield. First, a significant impairment occurred in the stationary eye conditions, attesting for an alteration of visuo-spatial encoding. Second, in the saccade conditions, an additional perceptual deficit (an error of $\sim 5^{\circ}$ along the saccade direction) was observed in both tasks and mainly in conditions where extra-retinal signals are thought to be critically involved, revealing a constant underestimation by extra-retinal signals of the saccade size, despite preserved saccade accuracy. These findings highlight a crucial role of the PPC in saccadic remapping processes underlying perceptual visual constancy and provide empirical evidence for models such as Ziesche and Hamker's (2014).
\end{abstract}

Keywords: corollary discharge, posterior parietal cortex, visual localization, remapping, transsaccadic integration 


\section{INTRODUCTION}

Sensing and interacting with our environment is one of the main brain functions. In primates the visuo-oculomotor system critically contributes to this function. A long-standing question for philosophers, psychologists and neurophysiologists is how can a subjective experience of stability of our visual environment emerge from ever-changing retinal images due to our own saccadic eye movements. This question translates into how retinal images from successive gaze fixations are integrated with signals related to the corresponding saccadic eye movements to achieve adequate visual perception. A long-held view is that such 'transsaccadic remapping' of visual information mainly involves extra-retinal signals derived from oculomotor commands ('efference copy': Von Holst \& Mittelstaedt, 1950, or 'corollary discharge': Sperry, 1950), with some secondary contribution of more sluggish proprioceptive signals sensing the actual ocular motion and position. Corollary discharge is thought to predictively encode the saccadic eye displacement and to interact with visual centers so as to cancel visual perturbation related both to the fast motion of retinal images during the saccade ('saccadic suppression') and to the retinal shift between the pre-saccadic and the postsaccadic images ('saccadic remapping'). In support of saccadic remapping, numerous behavioural studies (reviewed in Sommer \& Wurtz, 2008 ; Wurtz, 2008) measuring goaldirected motor responses (classic double step paradigm: Hallett \& Lightstone, 1976; Becker \& Jürgens, 1979; Mays \& Sparks, 1980; Goldberg \& Bruce, 1990) or perceptual localization judgments (Deubel, Schneider \& Bridgeman, 1996; Zimmerman \& Lappe, 2010), have demonstrated our ability to accurately localize spatial locations presented in temporal vicinity of a saccade. The objective of the current study is to better understand the crucial roles of the posterior parietal cortex (PPC) in the processing and maintenance of visuo-spatial information across saccades in humans.

Converging evidence (reviewed in Rao, Mayo \& Sommer, 2016) has suggested that saccadic remapping involves a widely distributed neural system, involving the fronto-parietal eye fields (FEF, PEF) as well as several areas in the occipito-temporal visual cortex and, subcortically, the thalamus and superior colliculus (SC). In the monkey, the pre-saccadic shift of receptive fields, initially discovered in the lateral intraparietal area (LIP, Duhamel, Colby \& Goldberg, 1992a), has then been found in the FEF (Umeno \& Goldberg, 1997), SC (Walker, Fitzgibbon \& Goldberg, 1995; Churan, Guitton \& Pack, 2012) and early visual cortical areas : V2, V3, V3a (Nakamura \& Colby, 2002) and V4 (Tolias et al., 2001). That such anticipatory 
shift of receptive fields could contribute to the saccadic remapping phenomenon has been supported by the observations that both such neural and behavioural phenomena are impaired when the SC-FEF pathway is altered through pharmacological inactivation of the mediodorsal (MD) thalamic nucleus (Sommer, 2002; Sommer \& Wurtz, 2008; Cavanaugh, Berman, Joiner \& Wurtz, 2016). These observations in the monkey also pointed out that this subcortical-cortical pathway could convey a corollary discharge signal. In humans, studies testing the effect of cerebral lesions in patients or of cortical perturbation through neurostimulation in healthy subjects globally confirmed the saccadic remapping network delineated in monkey. Indeed, impaired behavioural responses (motor or perceptual) in trans-saccadic localization tasks were observed in patients with a lesion affecting the MD thalamic nucleus (Gaymard, Rivaud \& Pierrot-Deseilligny, 1994; Bellebaum, Daum, Koch, Schwarz \& Hoffmann, 2005a; Ostendorf et al., 2010, 2013), the fronto-parietal cortex (Duhamel, Goldberg, Fitzgibbon, Sirigu \& Grafman, 1992b) or the PPC (Heide, Blankenburg, Zimmermann \& Kömpf, 1995; Khan et al., 2008; Pisella et al., 2011). Similar impairments were also reported in healthy subjects when neurostimulation is applied through TMS over the FEF (Ostendorf, Kilias \& Ploner, 2011) or the PPC (van Donkelaar \& Müri, 2002; Morris, Chambers \& Mattingley, 2007). In addition, neuroimaging studies revealed activity patterns consistent with saccadic remapping in the parietal cortex (Medendorp, Goltz, Vilis \& Crawford, 2003; Merriam, Genovese \& Colby, 2003; Pierce, Saj \& Vuilleumier, 2019) and in the occipital visual cortex (Knapen, Swisher, Tong \& Cavanagh, 2016; Podvalny et al., 2017, Zimmermann, Weidner, Abdollahi \& Fink, 2016).

More specifically, models of visuo-spatial localization across saccades have suggested that the PPC could play an important role in integrating visual and extra-retinal information in these remapping tasks (Ziesche \& Hamker, 2011, 2014; Ziesche, Bergelt, Deubel \& Hamker, 2017). Therefore, the PPC would be the core of the network involved in saccadic remapping. However, several questions remain as to the exact role of the PPC. First, Rath-Wilson and Guitton (2015a) showed largely intact efference copy functioning in parietal patients by changing the temporal sequence and duration of the two visual targets. This highlighted that the initial target location encoding process should be disentangled from the subsequent saccadic remapping process. Second, most of the causal evidence come from the effects of PPC perturbation in humans on motor manifestation of saccadic remapping such as in the double saccade task (patients: Duhamel et al., 1992b; Heide et al., 1995; Pisella et al., 2011; healthy subjects: van Donkelaar \& Müri 2002; Morris et al., 2007). In comparison, only few 
studies assessed a role of PPC in perceptual manifestation of saccadic remapping. Using TMS in healthy subjects, both Chang \& Ro (2007) and Collins \& Jacquet (2018) interpreted their TMS-induced deficit observed in a saccadic suppression of displacement (SSD) task as resulting from an impaired integration of retinal and extra-retinal signals (but see Fabius, Nijboer, Fracasso \& Van der Stigchel, 2020 and Ten Brink, Fabius, Weaver, Nijboer \& Van der Stigchel, 2019 parietal patients studies), whereas deficits disclosed by Prime, Vesia and Crawford (2008) in a task of transsaccadic comparison of visual features led them to propose a role of the PPC in spatial transsaccadic memory. This latter proposal echoes the frequently suggested deficit of transsaccadic spatial working memory in the neglect literature (for reviews, see Pisella \& Mattingley, 2004 and Pisella et al., 2011). Note however that the metric of spatial errors in these perceptual tasks have not been assessed, which thus did not allow to argue on the involvement of altered extra-retinal signals. More generally, the lack of solid behavioural evidence measured in perceptual tasks for a PPC involvement in saccadic remapping does not fit with the view, which is suggested by empirical data on (oculo)motor tasks and by theoretical models, of a major integrating role of the PPC between extra-retinal signals and spatial visual information. Further, the mere contribution of the cerebral cortex in processing extra-retinal signals for contraversive saccades has been challenged by RathWilson and Guitton (2015b) who revealed that patients with complete hemispherectomy adequately perform double step saccades requiring transsaccadic remapping. Finally, besides in Prime et al. (2008), previous studies did not evaluate the patients' behaviour in a baseline condition without eye movement to isolate possible deficits related to any PPC role in spatial visual processing independent of eye movements.

Therefore, the main aim of the current study was to assess the role of the PPC in saccadic remapping, and to distinguish the PPC contribution in spatial visual processing from that in extra-retinal signals processing. We had the opportunity to test a patient who manifested optic ataxia in his left hemifield without sign of neglect, in two perceptual localization tasks performed with versus without an intervening saccade. We used the SSD task (Ostendorf et al., 2010) and the peri-saccadic flash localization task (Zimmermann \& Lappe, 2010), each one tested in the classic saccade condition and adapted in a baseline eye stationary condition. Importantly, in the literature, the SSD task is commonly divided in two versions ('GAP' and 'STEP') which are compared to test the ocular extra-retinal signals integrity (Deubel et al., 1996; Ostendorf et al., 2010). Indeed, previous studies in healthy subjects showed that intrasaccadic target jumps are more easily detectable when a gap of $250 \mathrm{~ms}$ breaks the 
constancy of the visual environment and yields the central nervous system (CNS) to more strongly rely on monitoring of extra-retinal signals ("blanking effect" Deubel et al., 1996; Ostendorf et al., 2010). The same kind of processing of extra-retinal signals is expected to occur in the peri-saccadic flash localization task because (1) the bar is flashed for a short duration during the latency of the saccade and (2) the saccade target is extinguished during the saccade, leading the CNS to use the information conveyed by the extra-retinal signals to infer the position of the bar.

\section{METHODS}

In this section, we report how we determined our sample size, all data exclusions, all inclusion/exclusion criteria, whether inclusion/exclusion criteria were established prior to data analysis, all manipulations, and all measures in the study.

\subsection{Participants}

Patient CF suffered in 2003 from a cerebral angeitis initially presenting with headache without fever. One week later, the patient was moderately confused with Balint's syndrome in absence of any purely motor, somatosensory or visual deficits (good visual acuity and binocular vision, no visual field defect, good object recognition and color perception). Cerebral angiography then showed vasospasm in the left middle cerebral artery territory and in the right posterior cerebral artery territory. MRI scans (figure 1) showed a posterior watershed infarct (ischemic lesion of junctional territories) resulting in distributed and asymmetrical bilateral lesions of the occipito-parietal region with a minute extension to the centrum semiovale (Brodmann's areas (BA) 18, 19, 7, 5, and 2). None of the laboratory studies provided a clear etiology either in terms of inflammatory or autoimmune disease. During the following months, simultanagnosia and neglect rapidly disappeared leaving the patient with a stable and isolated optic ataxia in his left visual hemifield without expression of right-sided symptoms (e.g. Khan et al., 2005a, 2008; Khan, Pisella \& Blohm, 2013; Blangero et al., 2008; Granek, Pisella, Blangero, Rossetti \& Sergio, 2012), thought to be the consequence of both larger damage of BA 7 in the right hemisphere and full sparing of the intraparietal sulcus (IPS) in the left hemisphere (see Figure 1). At the time of testing (April 
2018), patient CF was 41 years old and left unilateral optic ataxia was the sole persistent deficit.

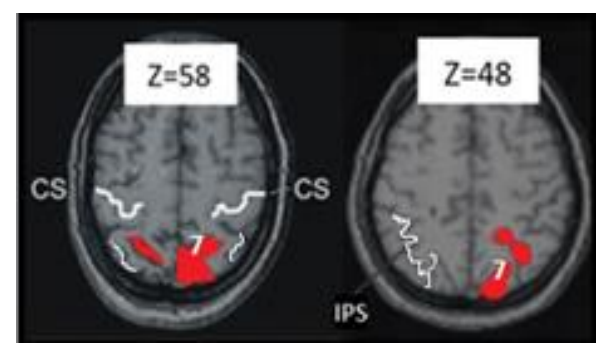

Figure 1: Patient CF's anatomical MRI brain slices under T2 contrast. The MNI z-coordinates of the axial slices are indicated. Lesions were mapped and colored in red. White numbers indicate damaged Brodmann' area 7 in the right hemisphere. IPS: intra-parietal sulcus, CS: central sulcus.

Four healthy subjects $(37.5 \pm 4.12$ years, one female) with normal or corrected-to-normal vision and no known neurological disease served as controls. The protocol was conducted in accordance with the ethical standards prescribed in the 1964 Helsinki Declaration and subsequent amendments to the declaration. All participants gave their informed consent.

\subsection{Visual stimuli}

The participants were seated in a dark room in front of a 19 inches CRT monitor (resolution: 1280 x 1024 pixels; vertical frequency: $85 \mathrm{~Hz}$ ) at a viewing distance of $57 \mathrm{~cm}$. To avoid visual landmarks or references (e.g., screen border) in the localization tasks (Awater \& Lappe, 2006; Deubel, Schneider \& Bridgeman, 2002) the monitor was covered with a neutral-density filter (ND4; transmittance $=25 \%$ ). In all tasks, the fixation points and saccadic targets were red circles $1^{\circ}$ in diameter presented on a dark background. The to-be-localized flash stimulus in the localization task (see below) was a vertical green bar, $0.15^{\circ}$ wide and $9^{\circ}$ high.

\subsection{Experimental procedure}

The experiment included three main tasks: a peri-saccadic flash localization task (LOC) and a saccadic suppression of displacement (SSD) task with a target jump with no delay (STEP version) and with a $250 \mathrm{~ms}$ delay (GAP version). Each of them has been performed in two conditions: either coupled with a saccade or under gaze fixation. Both visual hemifields have been tested in two sessions separated by a break in illuminated environment (see Table 1). A training of 5 trials preceded each condition of each task. Note that procedure and data of this 
study have been archived in a publicly accessible repository (https://osf.io/tdqxw/?view_only=022094ca59524d43a11b114e76c7e66b). The following section outlines each task for the left hemifield assessment (positions of stimuli were reversed for the right visual hemifield testing).

\begin{tabular}{|c|c|c|c|c|c|c|c|c|c|c|c|c|}
\hline \multicolumn{4}{|c|}{ LEFT VISUAL HEMIFIELD ASSESSMENT } & & \multicolumn{5}{|c|}{ RIGHT VISUAL HEMIFIELD ASSESSMENT } \\
\hline GAP & STEP & GAP & STEP & LOC & LOC & & GAP & STEP & GAP & STEP & LOC & LOC \\
SAC & SAC & FIX & FIX & SAC & FIX & & SAC & SAC & FIX & FIX & SAC & FIX \\
72 & 72 & 72 & 72 & 60 & 60 & & 72 & 72 & 72 & 72 & 60 & 60 \\
trials & trials & trials & trials & trials & trials & & trials & trials & trials & trials & trials & trials \\
\hline
\end{tabular}

Table 1. Experimental design. The two hemifields were tested in two sessions separated by a break. Each of the 3 tasks of a session (GAP version of SSD, STEP version of SSD, and LOC) was performed in a fixation condition (FIX) and a saccade condition (SAC). Each SSD block contained 72 trials (i.e., 3 staircases of 24 trials) and each LOC block included 60 trials (6 trials per bar position).

\subsubsection{Saccadic suppression of displacement task}

In the saccade condition, a fixation point was presented at 6 or $8^{\circ}$ to the right of the centre of the screen. Correct gaze fixation was ensured by monitoring gaze position online: fixation was validated as soon as the participant's gaze had remained for a variable period (from 800 to $1200 \mathrm{~ms}$ ) inside a nonvisible square ( $5^{\circ}$ sides) centred on the fixation point ('FP box'). Then, the fixation point was replaced by a saccade target (T1) at 6 or $8^{\circ}$ to the left of the centre of the screen. Such random variations of stimuli positions were intended to prevent stereotypic oculomotor behaviour. When the saccade was detected, the target jumped directly ('STEP-SAC' version) or after a blank of $250 \mathrm{~ms}$ ('GAP-SAC' version) at an unpredictable position (T2) (see figure $2 a$ and $2 b$ ). Participants were asked to manually press one of two keys to indicate whether the target had been displaced to the right or to the left from its initial position in a forced-choice manner (choosing randomly one of the two keys in case they are unsure). Target T2 switched off as soon as the participant's response was produced. The size of the jump was adapted by three independent, randomly interleaved staircases of 24 trials. Each staircase followed a one-up one-down logic with a constant step size of $1.5^{\circ}$ starting from an initial target jump of $3.5^{\circ}$ leftward, $3.5^{\circ}$ rightward and $0^{\circ}$ (no jump), respectively. This sequence of events was the same when the SSD tasks were performed within the right visual field but with all spatial parameter symmetrically reversed. 
In the fixation condition, the sequence of events was identical to that of the 'STEP-SAC' and 'GAP-SAC' versions just described (respectively for a version with a direct T1-to-T2 target jump named 'STEP-FIX' and a version with $250 \mathrm{~ms}$ gap between T1 disappearance and T2 appearance named 'GAP-FIX'), except for the saccade that was not produced, as participants were instructed to keep their gaze inside the nonvisible 'FP box' during the whole trial ; also the duration of initial target (T1) was fixed to $200 \mathrm{~ms}$. 

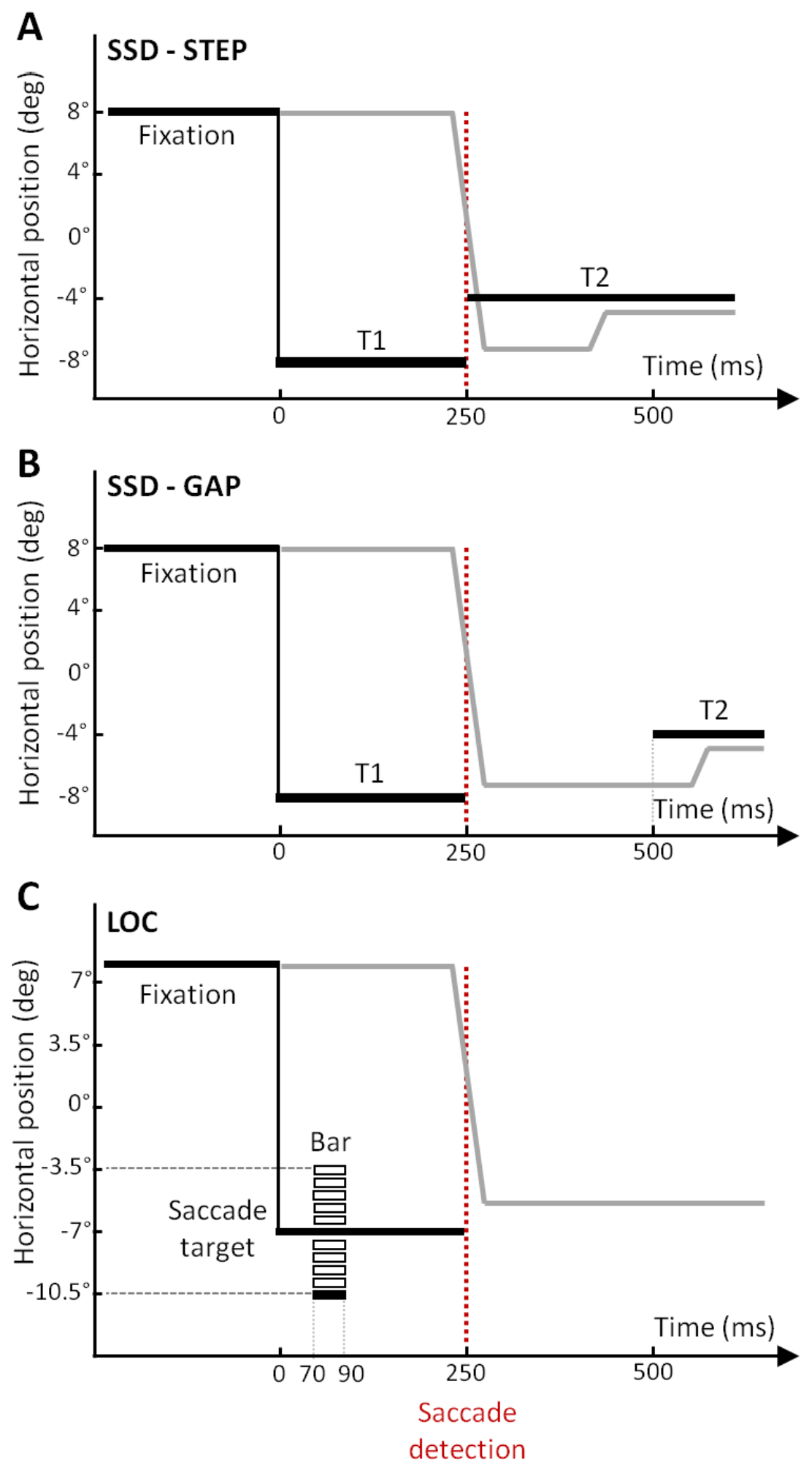

Figure 2. Schematics of the three tasks in the saccade condition. Time-course of the location of the different visual stimuli (bold black lines) and of gaze position (bold grey lines). A: SSD-task in STEP version. After a period of fixation, the participant executes a saccade toward a target (T1) that jumps during the saccadic response toward another position (T2). The participant must report the direction of the target jump by pressing one of two keys. B: SSD-task in GAP version. Same sequence of events as in the SSD - STEP task except that a $250 \mathrm{~ms}$ delay was introduced between the disappearance of T1 and the appearance of T2. C: Localization task. A saccade target is presented, followed $70 \mathrm{~ms}$ later by 
a bar briefly flashed $(20 \mathrm{~ms})$ randomly at one of 10 possible locations. After executing the saccade, the participant must report the spatial position of the bar by adjusting the location of a mouse pointer.

\subsubsection{Localization tasks}

In the saccade condition ('LOC-SAC'), trials began with a fixation point which appeared $8^{\circ}$ to the right of the centre of the screen for a random period (from 750 to $1250 \mathrm{~ms}$ ). Correct gaze fixation was ensured in the same way as described above. After this delay, the fixation point was switched off and a saccade target appeared $7^{\circ}$ to the left of the centre of the screen. Seventy milliseconds after the display of the target, a bar was flashed for $20 \mathrm{~ms}$ at a randomly chosen position among 10 positions around the target (see figure $2 c$ ). This timing of flash presentation relative to the reactive saccade execution (i.e. $~ 160 \mathrm{~ms}$, depending on saccade latency) has been chosen to avoid any other peri-saccadic mislocalizations that occur for stimuli flashed within 50 - $100 \mathrm{~ms}$ around saccade onset (Honda, 1991; Morrone, Ross \& Burr, 1997). If gaze position did not remain inside the nonvisible 'FP box' until the disappearance of the flash, the trial was considered as a failure and re-run at the end of the task. Then, the saccade was detected based on velocity and acceleration thresholds (see following section) and a black screen appeared at the eye movement onset for a duration of $850 \mathrm{~ms}$. This was followed by the appearance of a pointer at a random position near the bottom right corner of the screen. The participant had to move the pointer and to click at the position where he (she) had perceived the flashed bar or, but only in case he(she) did not see the flashed bar or was too unsure, to click near the bottom border of the screen. Again, the trial was not accepted and re-run later if the participant's gaze at the time of the mouse click was not inside a rectangular box adjacent to the 'FP box' and enclosing the flash possible locations (height: $5^{\circ}$, width: from the $5.5^{\circ}$ right location up to the left border of the screen). This sequence of events was the same when the LOC-SAC task was performed within the right visual field but with all spatial parameter symmetrically reversed.

In the fixation condition ('LOC-FIX'), the sequence of events was identical, except that no saccadic target was presented, and the participant had to maintain its gaze inside the nonvisible 'FP box' during the whole trial. Also, the duration of the black screen was increased by $160 \mathrm{~ms}$, matching the mean delay between bar disappearance and saccade onset in the LOC-SAC condition.

\subsection{Data analysis}




\subsubsection{Eye movement measurement}

Eye movements were continuously recorded (sampling frequency: $500 \mathrm{~Hz}$ ) with the Eyelink 1000 system in the Tower Mount configuration (SR Research, Ltd., Canada). The start and end of saccades were detected online using eye velocity and eye acceleration thresholds of $22 \% \mathrm{~s}$ and $4000 \% \mathrm{~s}^{2}$. Data stored on the recording PC were analysed off-line using a homemade Matlab routine and Data Viewer software (SR Research, Ltd., Canada). Trials in which the latency or the amplitude exceeded the mean plus or minus two standard deviations were excluded. Statistical comparisons between patient CF and the control group were performed by using the Crawford bilateral $t$-test for single cases (Crawford \& Garthwaite, 2002) since no systematic saccadic impairment could be predicted in optic ataxia (Gaveau et al. 2008).

\subsubsection{Saccadic suppression of displacement tasks analysis}

For each of the 5 subjects and 8 combinations of conditions (left/right hemifield x saccade/nosaccade x GAP/STEP), the psychometric function of participant's behavioural responses was determined by using psignifit, a toolbox implemented in Matlab. The same parameters as described in Ostendorf et al. (2011) were extracted from the psychometric curves: the point of subjective target stationarity (PSS) (= the target jump size yielding a 50\% proportion of forward reports) quantified the average performance (bias) of each participant and the slope of the psychometric curve assessed the uncertainty of the subject's perceptual responses. Statistical comparisons were performed by unilateral Crawford $t$-tests based on the following predictions: visual underestimation is strongly expected in the fixation conditions in the ataxic hemifield (Blangero et al., 2010 ; Vindras et al., 2016) and, in the saccade conditions, a shift of visual percept in the saccade direction is predicted from numerous studies (Ostendorf et al., 2010, 2011).

\subsubsection{Localization tasks analysis}

For each of the 5 subjects and 4 combinations of conditions (left/right hemifield x saccade/nosaccade), we extracted the horizontal (X) and vertical (Y) coordinates of the pointer response relative to the real position of the bar. Since the task involved only horizontal saccades, only the $\mathrm{X}$ coordinate of pointer position error was considered for statistical analyses. For each condition, the uncertainty of localization responses was assessed by averaging the variance of the reported responses across the 10 possible bar positions. Recall that trials in which there 
was an incorrect fixation of gaze were systematically re-run (see 'Experimental procedure' above). In the fixation condition, the mean percentage of re-run trials in patient $\mathrm{CF}(18.33 \%)$ differed significantly from those of the healthy group $(10.63 \% \pm 5.46$; one tailed Crawford $t$ test $: \mathrm{t}=4.68 ; \mathrm{p}<.01)$ while in the saccade condition, this percentage in $\mathrm{CF}(10.83 \%)$ and in healthy group $(15 \% \pm 6.87)$ did not differ significantly (one tailed Crawford $t$-test $: \mathrm{t}=-0.54$; $\mathrm{p}>0.05$ ). Note that we considered that our healthy participants are representative of the population and that our low sample size is nonetheless sufficient for the statistical comparisons with patient $\mathrm{CF}$. Indeed, for the localization tasks, the performance of these four control subjects did not differ significantly from those recorded in a previous (unpublished) study conducted in our laboratory with 30 healthy participants. In addition, for the SSD tasks, the psychometric curves obtained in the healthy subjects of the current study are very consistent and also disclose key characteristics (including changes between the STEP and GAP conditions) already reported in the literature (see Deubel et al., 1996 for an example).

\section{RESULTS}

\subsection{Asymmetry between visual hemifields under fixation condition}

The fixation condition was entered into the experiment to verify the presence of a perceptual deficit per se in CF, as suggested by a previous study using TMS over the PPC in healthy volunteers (Prime et al., 2008). A deficit under the stationary eye condition in our patient could therefore be a confounding factor which must be considered to isolate the pure contribution of extra-retinal signals in the trans-saccadic localization tasks. Raw results of the fixation condition are presented in figure 3 (SSD tasks; see also Table 2) and figure 4 (LOC task). 

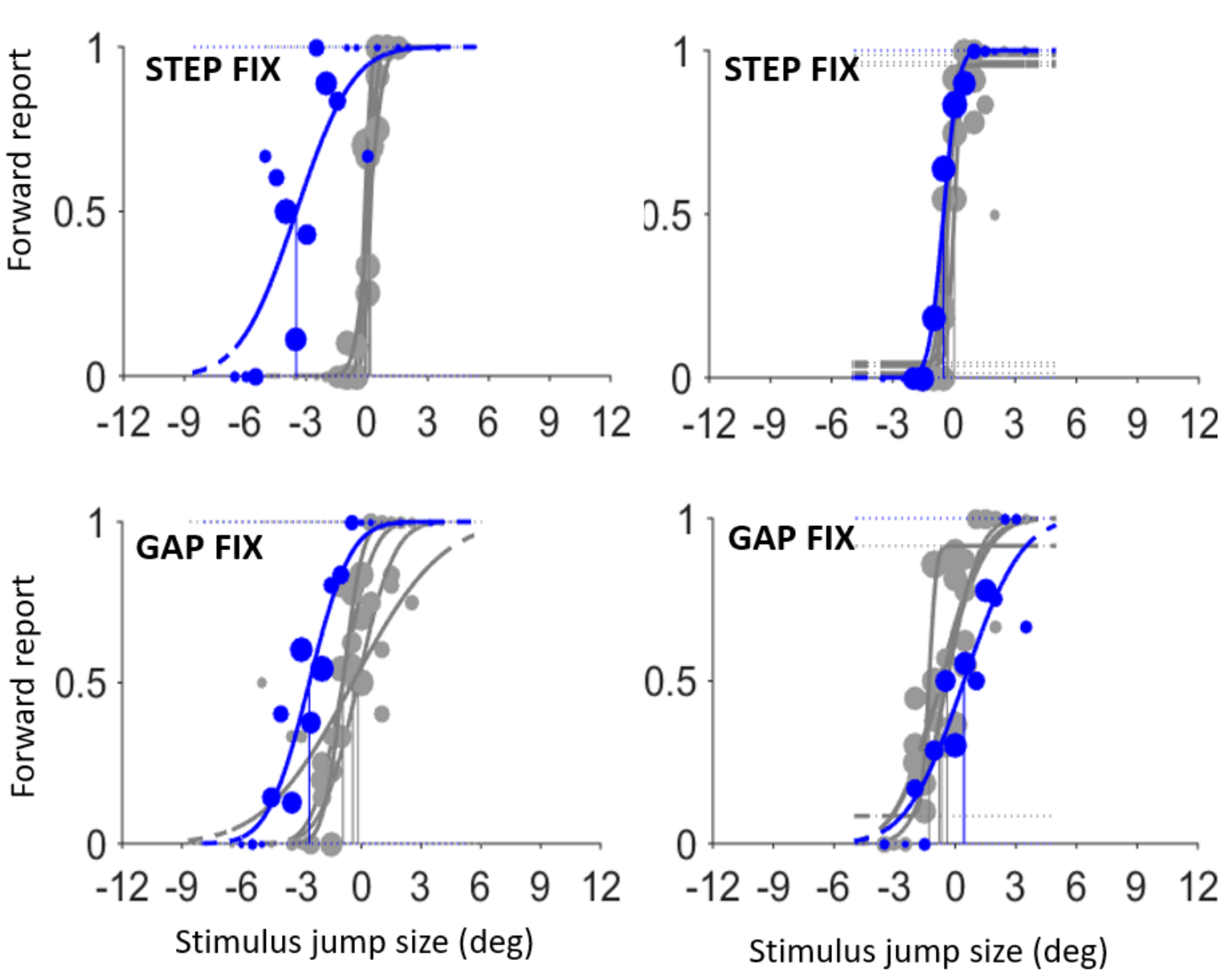

Figure 3. SSD tasks raw data in the fixation condition plotted separately for both hemifields: psychometric functions in patient $C F$ (blue lines and dots) and control subjects (grey lines and dots) fitted for the STEP-FIX (top) and GAP-FIX (bottom) conditions. The proportion of trials in which subjects reported an apparent stimulus jump in the saccade direction (forward) is plotted against the size of stimulus jump (positive $=$ forward; negative $=$ backward). Vertical lines (blue in CF, grey in controls) denote the $50 \%$ perceptual threshold or point of subjective stationarity - PSS (jump size eliciting backward and forward reports equally frequently); size of the dots indicates the number of trials for each target jump value. 

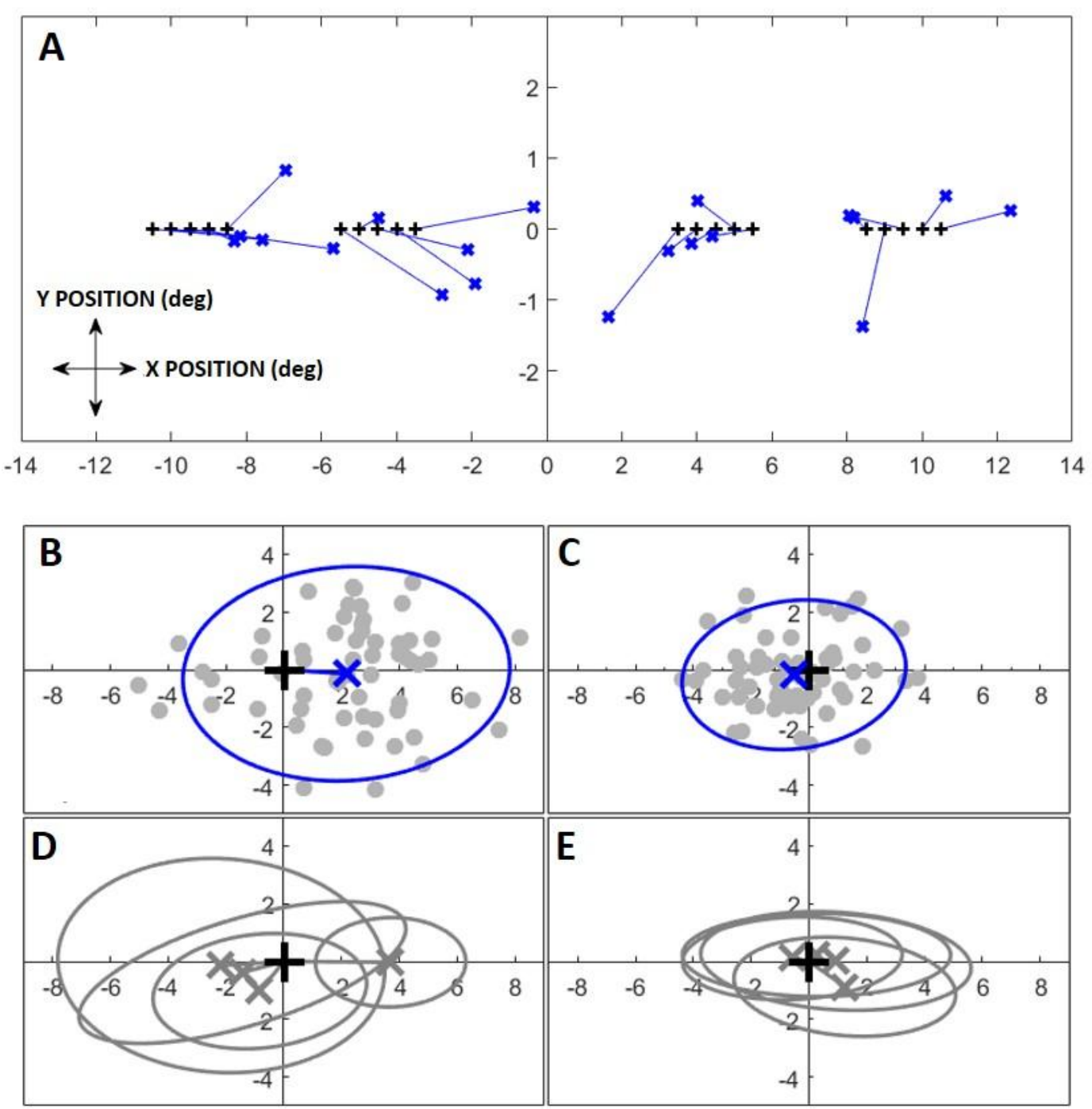

Figure 4. LOC task raw data in the fixation condition plotted separately for both hemifields. A: Mean 2-D localization errors in patient $C F$ for each bar position (black cross) depicted as error vectors (blue lines). Negative (positive) errors in the left hemifield and positive (negative) errors in the right hemifield correspond to overestimation (underestimation) of the visual target. B-E: Scatter of 2-D localization errors in patient $C F(\boldsymbol{B}-\boldsymbol{C})$ and control subjects $(\boldsymbol{D}-\boldsymbol{E})$ plotted after normalization of bar positions along the $X$-axis (normalized bar position = black ' + '). The mean localization error (blue or grey cross for $C F$ and controls, respectively) and the corresponding distributions of individual responses (90\% confidence interval, blue or grey ellipses) are shown for each subject. Grey dots in B$C$ show individual responses of patient $C F$.

To estimate whether the performance of the patient in his ataxic (left) hemifield was reduced as compared to that in the right hemifield, the difference of the perceptual responses between hemifields was calculated as follows:

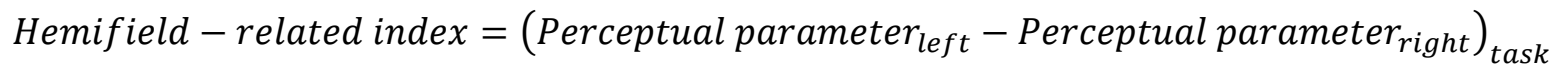


For the SSD tasks, we calculated for each condition (GAP and STEP) two hemifield-related indexes, respectively based on the perceptual bias and uncertainty (i.e., the PSS and slope of the fitted psychometric curves). As shown in figure 5, CF's hemifield-related indexes of bias obtained in the SSD tasks (STEP : $-2.97^{\circ}$; GAP : $-3.05^{\circ}$ ) lay outside the hemifield-related indexes of the control group $\left(0.27^{\circ} \pm 0.25^{\circ}(\mathrm{SD})\right.$ and $0.18^{\circ} \pm 0.3^{\circ}$, respectively), as confirmed statistically (STEP: $t=-11.59, \mathrm{p}<.01$; GAP: $t=-9.63, \mathrm{p}<.01$ ). Inversely, CF's hemifieldrelated indexes of uncertainty of CF were near zero (STEP : $-0.52^{\circ}$; GAP : $0.06^{\circ}$ ), similar to those reported in the control group for both STEP $\left(0.05^{\circ} \pm 0.53 ; t=-0.96\right.$; NS) and GAP ($\left.0.17^{\circ} \pm 0.34 ; t=0.61 ; \mathrm{NS}\right)$ conditions.

In the LOC task, the hemifield-related indexes of bias and uncertainty were calculated by considering the mean and the variance of localization errors, respectively. As shown in figure 5, CF's hemifield-related index of bias $\left(-1.67^{\circ}\right)$ and those of the control group $\left(-0.18^{\circ} \pm 3.27^{\circ}\right)$ did not differ significantly $(t=-0.41, \mathrm{NS})$, nor did hemifield-related index of uncertainty (CF: 4.47 ; control group: $0.21 \pm 3.29$ ). Note that a deficit of $\mathrm{CF}$ in the localization task could have been hidden by the larger between-subject variability observed in the control group in this latter task as compared to the SSD-tasks (see figure 5), which might be linked to the type of behavioral response (free-choice in LOC task versus forced-choice in SSD tasks).

In sum, whereas the small values of the hemifield-related index of bias in the control group signaled a balanced performance between the two hemifields for all three tasks, the negative values in the patient indicated a reduced performance in the left hemifield relative to the right hemifield. This difference between patient $\mathrm{CF}$ and the control group reached statistical significance for the two SSD tasks. Moreover, the pathological bias of CF in the left visual hemifield in these latter tasks was not accompanied by a greater uncertainty of the perceived stimulus position. 


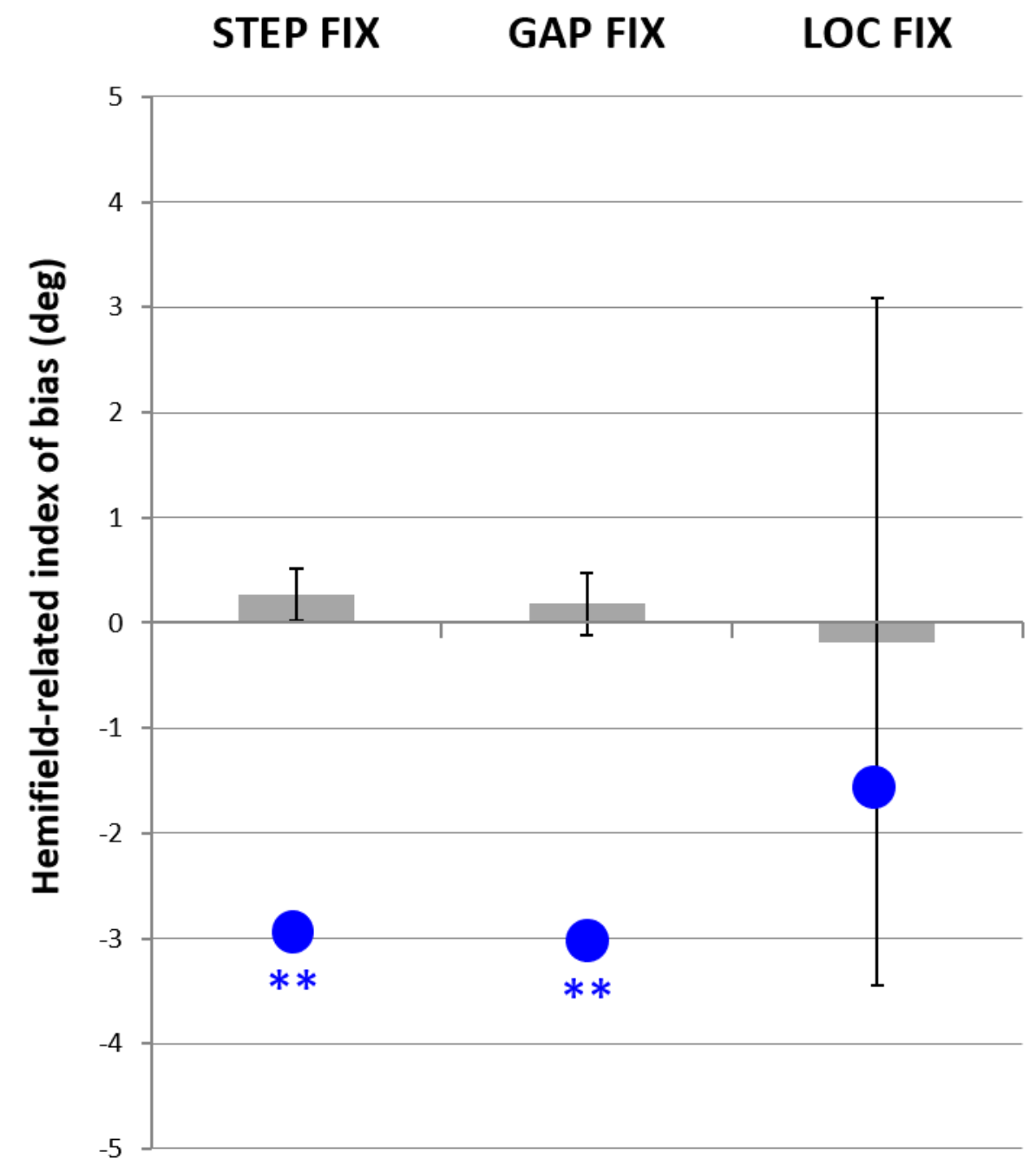

Figure 5: Hemifield-related indexes of bias in the fixation condition. Differences of mean performance between left and right visual hemifields for each of the 3 tasks in patient $C F$ (blue dots) and in the control group (grey bars and whiskers: mean +/- Standard Deviation). Statistical differences between $C F$ and control subjects (Crawford t test for single cases): ** $: p<.01$.

\subsection{Saccades versus fixation differences}

The raw results of the saccade condition are presented in figures 6 and 7 for the SSD and LOC tasks, respectively. Note that for the SSD tasks, we have reported in details the behaviour of participants in Table 2 . 
LEFT HEMIFIELD
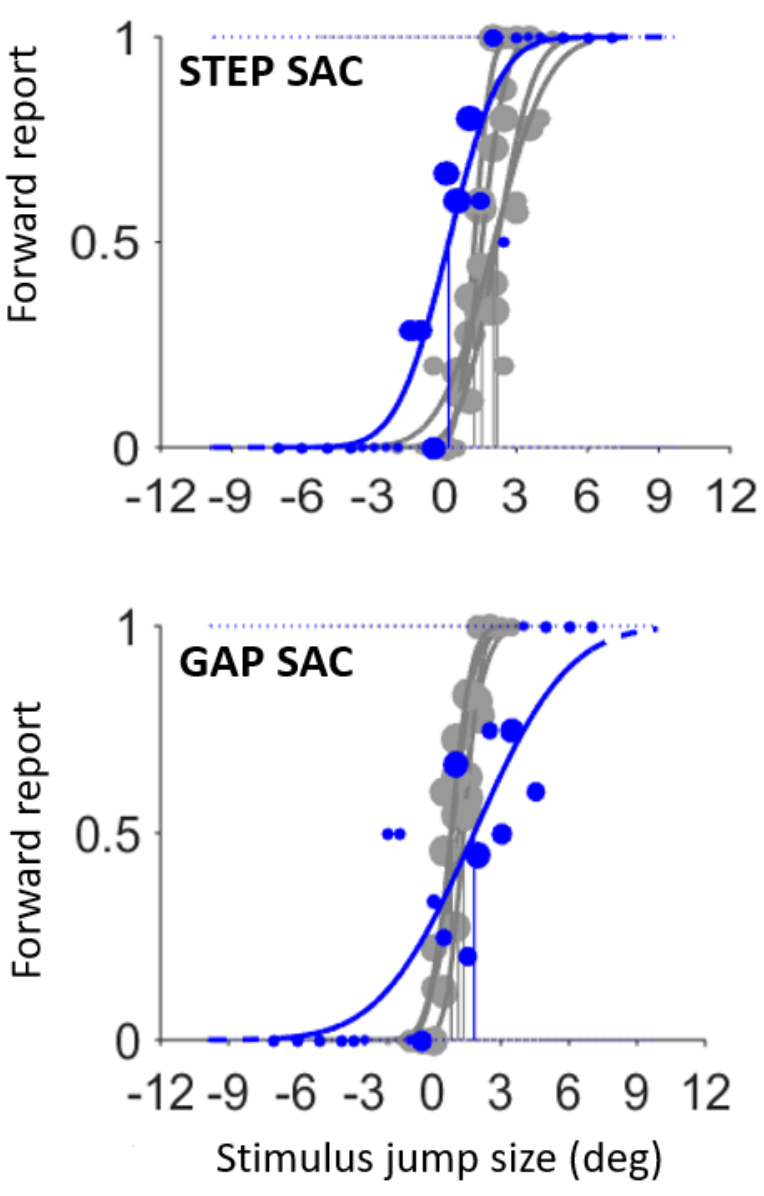

RIGHT HEMIFIELD
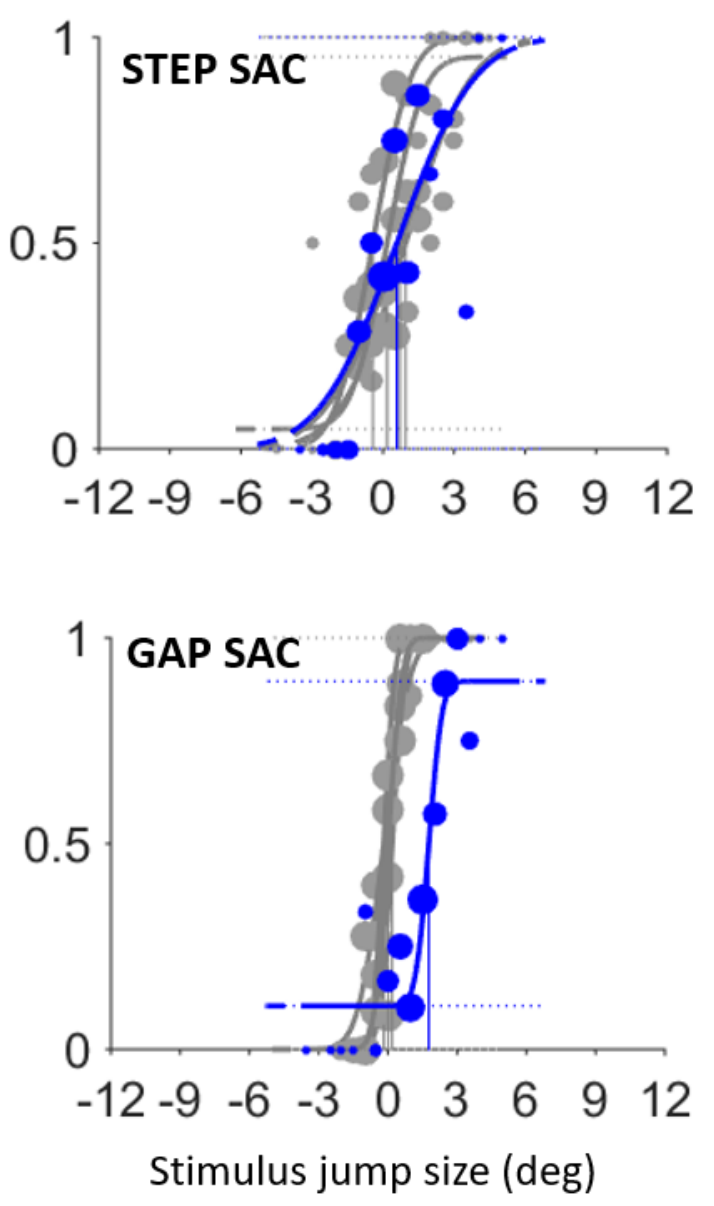

Figure 6. SSD tasks raw data in the saccade condition plotted separately for both hemifields: Psychometric functions in patient CF (blue lines) and control subjects (grey lines) fitted for the STEPSAC (top) and GAP-SAC (bottom) condition. Same conventions as in Figure 3. 

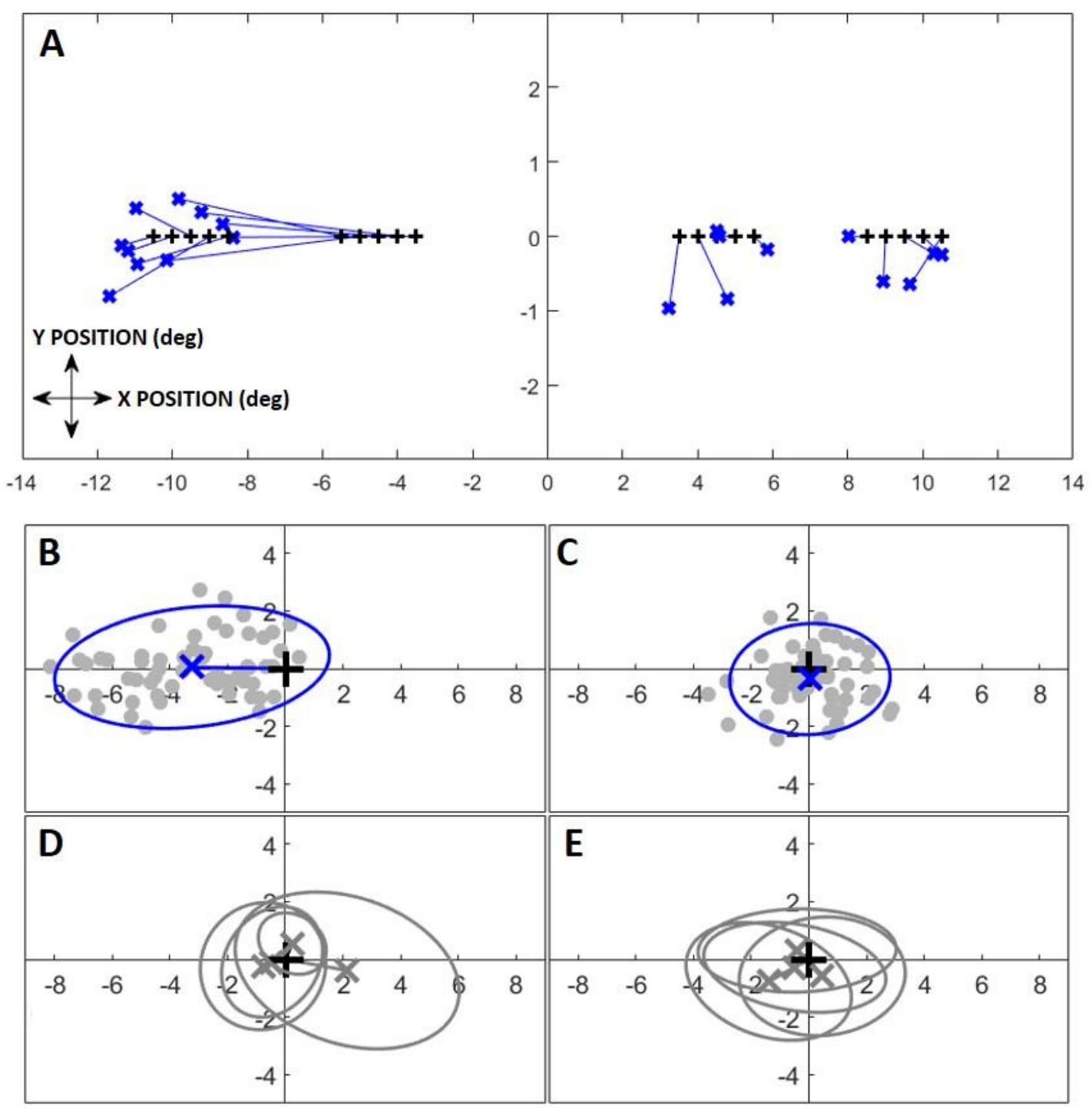

Figure 7. LOC task raw data in the saccade condition plotted separately for both hemifields. Same conventions as in Figure 4. 


\begin{tabular}{|c|c|c|c|c|c|c|}
\hline & \multicolumn{2}{|c|}{ GAP } & \multicolumn{2}{|c|}{ STEP } \\
\hline & & & SAC & FIX & SAC & FIX \\
\hline \multirow{4}{*}{$\mathrm{LH}$} & \multirow{2}{*}{ Bias } & CTL & $1.03( \pm 0.26)$ & $-0.62( \pm 0.38)$ & $1.75( \pm 0.45)$ & $0.04(0.12)$ \\
\hline & & $\mathrm{CF}$ & 1.82 & -2.62 & 0.14 & -3.48 \\
\hline & \multirow{2}{*}{ Slope } & CTL & $0.53( \pm 0.1)$ & $0.31( \pm 0.17)$ & $0.45( \pm 0.22)$ & $1.27( \pm 0.39)$ \\
\hline & & $\mathrm{CF}$ & 0.12 & 0.24 & 0.23 & 0.18 \\
\hline \multirow{4}{*}{$\mathrm{RH}$} & \multirow{2}{*}{ Bias } & CTL & $-0.04(0.18)$ & $-0.8( \pm 0.38)$ & $0.35( \pm 0.61)$ & $-0.24(0.17)$ \\
\hline & & CF & 1.77 & 0.43 & 0.58 & -0.51 \\
\hline & \multirow{2}{*}{ Slope } & CTL & $0.84( \pm 0.27)$ & $0.48( \pm 0.45)$ & $0.24( \pm 0.08)$ & $1.22( \pm 0.27)$ \\
\hline & & $\mathrm{CF}$ & 0.7 & 0.18 & 0.16 & 0.7 \\
\hline
\end{tabular}

Table 2. Bias and slope values during each condition of the SSD task in CF and control subjects (mean \pm standard deviation). LH : left hemifield ; RH : right hemifield ; CTL : control subjects.

Given that we reported above a deficit under the fixation condition in $\mathrm{CF}$, we calculated a saccade-related index reflecting the pure effect related to saccade execution by substracting, for a given task and in a given hemifield, the performance measured in the fixation condition from that measured in the saccade condition (figure 8):

Saccade - related index $=\left(\text { Perceptual }_{\text {parameter }} \text { sac }_{-}-\text {Perceptual parameter }_{\text {fix }}\right)_{\text {task }}$

Again, in each of the three tasks, two indexes were computed: a saccade-related index of bias and a saccade-related index of uncertainty were respectively obtained from the PSS and slope of the fitted psychometric curves (SSD tasks) or from the constant and variable localization errors (LOC task).

\subsubsection{Right (non ataxic) visual hemifield assessment}

In the case of rightward saccades, CF performed quite similarly to control subjects (figure 8). Firstly, in the SSD-tasks, CF's saccade-related indexes of bias (STEP : $1.09^{\circ}$; GAP : $1.34^{\circ}$ ) did not differ significantly from those reported in the control group in both STEP $\left(0.59^{\circ} \pm\right.$ $0.52 ; t<1$, NS) and GAP $\left(0.76^{\circ} \pm 0.46 ; t=1.13\right.$, NS $)$ conditions. Similarly, the saccaderelated indexes of uncertainty of CF were close to 0 (STEP : -0.54; GAP : 0.52) and did not differ significantly from those reported in the control group in each STEP $(-0.98 \pm 0.32 ; t=$ 1.23 , NS) and GAP $(0.35 \pm 0.49 ; t=0.31$, NS $)$ condition. Concerning the oculomotor 
behavior, we analyzed saccade latency and amplitude in the STEP and GAP conditions of SSD separately for the three target eccentricities $\left(12^{\circ}, 14^{\circ}\right.$ and $16^{\circ}$, see figure 9$)$. In the STEPSAC condition, saccade amplitudes of CF $\left(12.26^{\circ} ; 13.89^{\circ}\right.$ and $16^{\circ}$ respectively) globally remained within the performance range of the control group $\left(10.94^{\circ} \pm 1.28 ; 12.63^{\circ} \pm 1.34\right.$ and $14.54^{\circ} \pm 1.53$ respectively), as confirmed by the lack of statistical differences $(t<1$ for each comparison). Likewise, the latency of saccades performed by $\mathrm{CF}$ for each target eccentricity in this condition (207.27 ms ; $230.09 \mathrm{~ms}$ and $235.25 \mathrm{~ms}$, respectively) did not differ significantly from the control group values $(209.43 \pm 14.84 ; 211.88 \pm 14.22$ and $208.78 \pm$ 12.16, respectively). Analyses of saccade amplitude carried out in the GAP-SAC condition also did not reveal any difference between the primary saccades of CF $\left(10.98^{\circ} ; 12.16^{\circ}\right.$ and $13.63^{\circ}$ respectively) and those of the control group $\left(11.25^{\circ} \pm 1.71 ; 13.33^{\circ} \pm 2.02\right.$ and $15.21^{\circ}$ \pm 2.22 respectively, $t$-values $<1$ for each comparison). The latency of saccades performed by $\mathrm{CF}$ in this GAP-SAC condition was very similar to that of healthy participants for the $12^{\circ}$ target eccentricity (CF : $229.13 \mathrm{~ms}$; control group : $206.74 \pm 8.69 \mathrm{~ms}$ ) but, as the target eccentricity increased to $14^{\circ}$ and $16^{\circ}$, the latency of $\mathrm{CF}$ became longer ( $245.45 \mathrm{~ms}$ and 250 $\mathrm{ms})$ as compared to the control group $(197.36 \pm 4.7 \mathrm{~ms}$ and $202.86 \pm 11.92 \mathrm{~ms} ; t=9.15 ; \mathrm{p}<$ .01 and $t=3.54 ; \mathrm{p}<.05$, respectively). Note that a longer saccade latency in the SSD tasks is not critical for interpreting the subsequent perceptual behavior since the to-be-localized stimulus is precisely the saccade target (which stays visible on the screen until saccade initiation).

Secondly, in the LOC task, CF's perceptual behavior was also close to that of healthy participants (figure 7, top right panel, and Figure 8): the saccade-related index of CF's bias was near zero $\left(0.56^{\circ}\right)$ and did not differ significantly from that of the control group $\left(-0.45^{\circ} \pm\right.$ $0.96 ; t<1, \mathrm{NS})$. Also, concerning the saccade-related index of uncertainty, no statistical difference between CF's index (-0.96) and those of the control group (-2.43 \pm 0.96$)$ was reported $(t=1.37$; NS). Concerning the oculomotor behavior, CF generated saccades quite similar to those of the control group in terms of amplitude ( $\mathrm{CF}: 13.92^{\circ}$; control group : $14.86^{\circ} \pm 0.75$; NS) but not in terms of latency (CF : $294.46 \mathrm{~ms}$; control group : $205 \pm$ $13.21 \mathrm{~ms} ; t=6.06, \mathrm{p}<.01)$.

In sum, the visual perception behavior of CF was similar to that of the control group in terms of bias and uncertainty in the right visual hemifield. Also, his oculomotor behavior was similar to the control behavior in terms of saccade amplitude while differed slightly from control values in terms of latency. Thus, this patient is able to integrate extra-retinal signals to 
predict accurately the consequences of its own oculomotor actions in its right (non ataxic) hemifield.

\subsubsection{Left (ataxic) visual hemifield assessment}

In the case of leftward saccades, the pattern of CF behavior was dramatically different from that of the control group (figure 8).
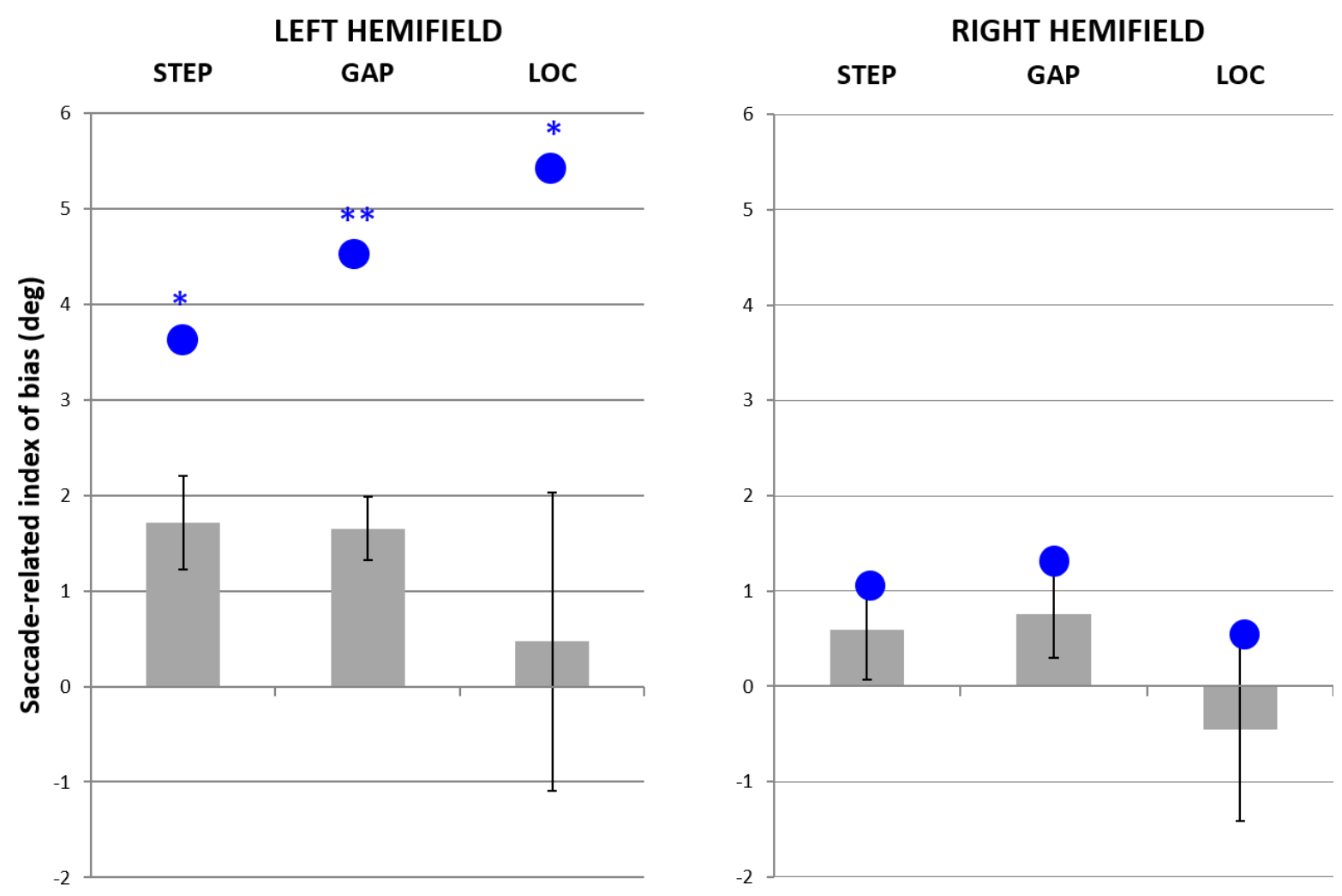

Figure 8. Saccade-related index of performance bias. Differences of mean performance between saccade and fixation conditions for each of the three tasks and the two hemifields in patient CF (blue dots) and in the control group (grey bars: means and whiskers: mean +/- Standard Deviation). Statistical differences between $C F$ and control subjects (Crawford t test for single cases): $*: p<.05$.

First, in the SSD STEP task (i.e., in which the contribution of extra-retinal signals is the lowest), the saccade-related index of bias was deteriorated in $\mathrm{CF}\left(3.62^{\circ}\right)$ and differed significantly from that of the control group $\left(1.71 \pm 0.49^{\circ} ; t=3.49 ; \mathrm{p}<.05\right)$. Importantly, this deficit could not be explained by other factors. Indeed, the saccade-related indexes of uncertainty in CF (0.05) and the control group $(-0.82 \pm 0.35)$ did not differ significantly $(t=$ 2.23 , NS). Also, the mean saccade amplitude of $\mathrm{CF}\left(-13.08^{\circ} ;-14.3^{\circ}\right.$ and $-16.03^{\circ}$ respectively 
for the $-12^{\circ},-14^{\circ}$ and $-16^{\circ}$ target eccentricities) globally fell in the control group ranges ($10.9^{\circ} \pm 1.47 ;-12.88^{\circ} \pm 1.79$ and $-14.49^{\circ} \pm 2.23^{\circ}$, respectively) with no statistical difference $(t$ values between -1.33 and 0 ). Finally, the latency of saccades to $12^{\circ}$ and $14^{\circ}$ targets in $\mathrm{CF}$

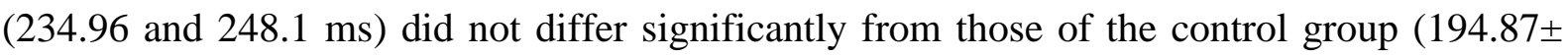
13.83 and $190.73 \pm 21.02 \mathrm{~ms}$, respectively) and only the latency of his saccades to the $16^{\circ}$ target significantly increased (CF : $250.78 \mathrm{~ms}$; control group : 195.61 $10.32 ; \mathrm{t}=4.78$, $\mathrm{p}<.05$ ), a pattern similar to that reported above for the right visual hemifield. In sum, neither the variability of the perceptual responses nor the oculomotor behavior could explain the deficit of CF. Rather, this shift of visual percept is mostly linked to gaze fixation, as seen by looking at the PSS values obtained in CF separately in the STEP-FIX and STEP-SAC conditions $\left(-3.48^{\circ}\right.$ versus $0.14^{\circ}$, respectively).

Second, in the SSD GAP task (i.e., in which the contribution of extra-retinal signals is the highest), the saccade-related index of CF's bias was drastically deteriorated $\left(4.44^{\circ}\right)$ and differed significantly from healthy subjects $(1.65 \pm 0.33 ; t=7.56 ; \mathrm{p}<.01)$. As previously, the saccade-related index of CF's uncertainty (-0.12) fell in the control range $(0.22 \pm 0.19 ; t=-$ 1.6, NS). In addition, the mean saccade amplitude of CF $\left(-11.95,-13.4\right.$ and $-14.51^{\circ}$, respectively for the $-12,-14$ and $-16^{\circ}$ targets) did not differ significantly from that of the control group $\left(-11.36^{\circ} \pm 1.33 ;-13.12^{\circ} \pm 1.34\right.$ and $-14.94^{\circ} \pm 1.78$, respectively) with $t$-values between -1 and 1 (see figure 9). Also, the mean saccade latency of CF for the three target eccentricities (222.35 ms, $241.63 \mathrm{~ms}$ and $235.22 \mathrm{~ms}$, respectively) did not significantly differ from those of the control group $(201.27 \pm 12.22 \mathrm{~ms}, 204.32 \pm 11.36 \mathrm{~ms}$ and $202.64 \pm 11.23$ ms). In sum, the deficit of CF could not be explained by differences in terms of a great variability of perceptual judgements within its left visual hemifield nor in terms of saccade execution. Regarding the raw data of PSS in the GAP-FIX and GAP-SAC conditions, the deficit of CF may be explained by a shift of the psychometric curves under gaze fixation ($\left.2.62^{\circ}\right)$ and, in the GAP-SAC condition, by an additional shift in the forward direction $\left(1.82^{\circ}\right)$. Indeed, the GAP-STEP difference of patient CF in the saccade condition (1.68 $\left.{ }^{\circ}\right)$ departs significantly from that of control subjects within the left hemifield $\left(-0.72^{\circ}\right.$; SD : 0.67) $(t=3.2$ ; $\mathrm{p}<.05)$, and not within the right hemifield $(t=1.71 ; \mathrm{NS})$. This pattern of results, echoing previous findings in thalamic patients, is consistent with an improper computation of extraretinal signals leading to an underestimating of the true saccade size (Ostendorf et al., 2010). This finding means that, in the left (ataxic) hemifield, while controls reported the direction of leftward displacement at chance level until it reached $1.65^{\circ}$ on average, $\mathrm{CF}$ needed a 
displacement $\sim 3^{\circ}$ larger than for controls to be able to begin to judge a physically leftward displacement as being leftward in more than $50 \%$ of the trials.

Third, in the LOC task, CF showed a strong deficit expressed by a systematic overestimation of the position of the bar (see figures $7 A$ and $7 B$ ). This was evidenced by a high saccaderelated index of constant error $\left(5.4^{\circ}\right)$ which differed significantly from that of the control group $\left(0.47^{\circ} \pm 1.56 ; t=2.83 ; \mathrm{p}<.05\right)$. As for the SSD tasks, no difference was reported between the saccade-related indexes of CF's uncertainty (-4.45) and those of the control group $(-2.45 \pm 3.53)$. Also, neither the amplitude nor the latency of saccades showed any difference between CF (mean amplitude: $-17.09^{\circ}$; mean latency: $273.36 \mathrm{~ms}$ ) and the control group $\left(-15.6 \pm 0.69^{\circ}\right.$ and $230.04 \pm 44.14 \mathrm{~ms}$; t-values of -1.93 and 0.88 , respectively). These findings are entirely consistent with the behavior of CF in the SSD-GAP task and corroborate the hypothesis of an improper computation of extra-retinal signals leading to an underestimation of the true saccade size. Indeed, the mean localization errors of CF computed separately in the LOC-FIX and the LOC-SAC conditions point toward opposite directions, respectively underestimating by $2.17^{\circ}$ and overestimating by $3.23^{\circ}$, the two adding together to yield, as in the SSD-GAP task, a large visual overestimation (i.e. a $5.4^{\circ}$ shift in the forward direction).

Altogether, the values of the saccade-related indexes of CF in the SSD-GAP task and in the LOC task were very similar $\left(4.44^{\circ}\right.$ and $5.4^{\circ}$, respectively). When referred to the actual mean saccade amplitudes of $\mathrm{CF}$ in these two tasks $\left(-13.29^{\circ}\right.$ and $-17.09^{\circ}$, respectively), these measures point to similar estimates of underestimation of the true saccade magnitude $(33.41 \%$ in the SSD-GAP task and $31.6 \%$ in the LOC task). In both cases, the direction of the saccaderelated indexes (i.e., overestimation in both tasks) revealed a forward shift of the predicted position of the to-be-localized stimuli (i.e., T2 in SSD-GAP task and the flash in the LOC task) from the actual eye position after saccade execution. 


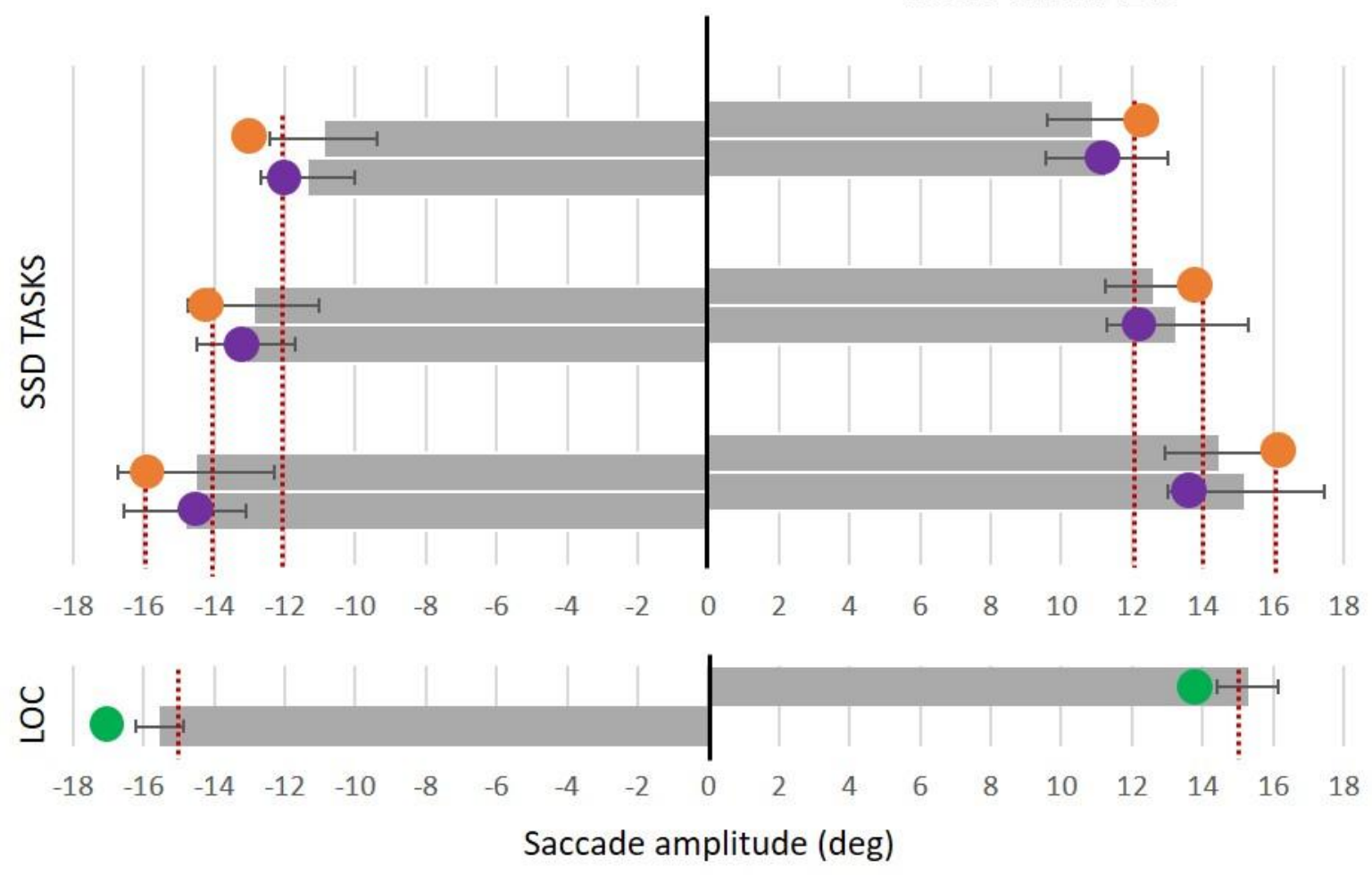

Figure 9. Amplitude of saccadic eye movements in the SSD tasks (top) and LOC task (bottom) plotted separately for both hemifields. Circles represent mean amplitude in CF (orange : STEP task; purple: GAP task; green: LOC task); grey bars represent mean amplitude in control subjects (+/- standard deviation). Vertical dashed lines indicate target eccentricity.

\section{DISCUSSION}

In the present case study, we aimed to assess the role of the PPC in saccadic remapping, and more precisely to determine the PPC relative contribution in spatial visual processing and extra-retinal signals processing. For this purpose, we tested patient $\mathrm{CF}$ and age-matched controls in a saccadic suppression of displacement task (GAP and STEP versions) and a perisaccadic localization task (LOC). These three tasks have been performed both in their original saccade condition and in their corresponding ocular fixation condition. Compared to healthy participants, patient CF showed a specific pattern of perceptual deficits in the ataxic (left) hemifield. First, a significant impairment occurred in the stationary eye conditions, pointing toward an alteration of spatial visual encoding. Second, in the saccade conditions, despite a globally preserved oculomotor behaviour, an additional perceptual deficit was observed in tasks where extra-retinal signals are thought to be critically involved i.e., SSD-GAP and LOC tasks. This second perceptual deficit was strongly consistent between these two tasks (a shift 
of $\sim 5^{\circ}$ target localization error in the forward direction) revealing a constant underestimation of the true saccade size in the remapping process. In the following sections, we discuss these two points and try to better characterize the role of the PPC within the neural circuitry known to underlie spatial remapping.

\subsection{A deficit in processing of visual information in eye fixation condition in the left hemifield}

As can be seen on figure 4, patient CF was largely and systematically hypometric when required to indicate with the computer mouse where a visual bar has been flashed within his left (ataxic) visual hemifield while maintaining eye position on the fixation point at $8^{\circ}$ to the right of the centre of the screen (LOC-FIX condition). This error pattern confirms a spatial encoding alteration expressed in an eye-centred reference frame (Khan et al., 2005a; Khan, Pisella, Rossetti, Vighetto \& Crawford, 2005b; Dijkerman et al., 2006) as centripetal errors toward the eye fixation position for visual targets presented in the contralesional hemifield (Gaveau et al., 2008; Blangero et al., 2010; Vindras et al., 2016). This mislocalization is known to be partially compensated for when more time is available for spatial encoding (Gaveau et al., 2008; Rossetti et al., 2005; Milner, Paulignan, Dijkerman, Michel \& Jeannerod, 1999; Milner, Dijkerman, McIntosh, Rossetti \& Pisella, 2003).

Patient CF was also impaired at judging the direction of target displacements within his left (ataxic) visual hemifield in the GAP and STEP fixation conditions. As can be seen on figure 3, whereas patient CF exhibited normal performance within the right hemifield (PSS near $0^{\circ}$, with a more flattened psychometric curve in GAP-FIX than STEP-FIX as in control subjects), his performance in the left hemifield was impaired in two aspects. First, the psychometric curve was shifted toward the left, revealing that small backward (rightward) displacements of the target within the left hemifield were perceived leftward. This deficit could be explained by an inverse relationship between the duration of the target and the hypometric bias of spatial encoding discussed in the previous paragraph. Indeed, being briefly flashed target T1 would be more underestimated than target $\mathrm{T} 2$ which remains visible until the subject's response, thus resulting in the observed decrease of the probability of backward reports in favour of forward reports. Second, in addition to this perceptual bias, the psychometric curve of patient CF appeared similarly flattened when the second target position was presented immediately as when it was presented after a $250 \mathrm{~ms}$ blank. This second aspect of CF's behaviour is different 
from what was observed in healthy controls, i.e. steeper psychometric curves in STEP relative to GAP. It is classical to observe in healthy participants with the eyes stationary that the immediate target displacement of the STEP condition, because it creates perceived apparent motion (phi phenomenon), reduces the uncertainty range of direction discrimination. Accordingly, impairment of high-level motion perception, including apparent motion (phi phenomenon) and biological movements identification, has been reported in patients with unilateral or bilateral parietal lesions (Batelli et al., 2001; Michel \& Henaff, 2004) in absence of deficit of low-level motion perception (motion perception based on colour contrast, recognition of motion-defined shapes, optic flow perception, as well as the waterfall illusion of motion after-effect). Michel and Henaff (2004) reported that patient AT with bilateral optic ataxia was seeing successive flashing lights when normal subjects would see apparent motion. This absence of phi phenomenon in both STEP and GAP conditions for patient CF could explain why the benefit of the STEP condition over the GAP condition classically observed in healthy subjects was not present in CF's left hemifield. This highlights the importance of subtracting the localization errors between the saccade and the fixation conditions to correctly interpret in patient $\mathrm{CF}$ the specific contribution of extra-retinal information in the SSD tasks. While the performance of patient $\mathrm{CF}$ in the bar localisation task and the first aspect of his deficit in the SSD task (i.e. the leftward shift of the psychometric curve) in the left hemifield can both be explained by the previously reported hypometric bias in spatial visual encoding within the ataxic field, this second aspect of patient CF's deficit remains mysterious. We speculate that it could be due to a larger, probably overlapping, spatial uncertainty range for both first and second target locations, explaining why the patient verbally reported to "see two grossly large spots of light successively flashed in his left peripheral vision without any precise localization information".

\subsection{A preserved role of extra-retinal signals in perceptual judgments within the healthy visual field}

When performing the trans-saccadic SSD tasks, healthy participants performed better in the GAP-SAC than in the STEP-SAC condition, irrespective of the tested visual hemifield (average threshold over both hemifields of $1.05^{\circ}$ in STEP-SAC condition and $0.5^{\circ}$ in the GAP-SAC condition). Indeed, the psychometric curves in the GAP-SAC condition show a $50 \%$ threshold closer to 0 and a slope steeper than in the STEP-SAC condition, for each 
healthy participant and each hemifield (see figure 6). Psychometric curves in patient CF do not show this so clearly (figure 6), probably due to the contamination of the perceptual deficit of apparent motion pre-existent in the left hemifield under the fixation condition, but the slope of the curve within his right visual hemifield is steeper in the GAP as compared to the STEP condition. This enhancement in the GAP-SAC relative to the STEP-SAC condition is a very consistent observation across studies and refers to the so-called "blanking effect" (Deubel et al., 1996; Collins, Rolfs, Deubel \& Cavanagh, 2009; Ostendorf et al., 2010, 2011). This effect indicates that the decision-making process leading to a trans-saccadic perceptual judgement may rely on different mechanisms depending on the available information at the end of the saccadic eye movement (Hamker, Zirnsak, Ziesche \& Lappe, 2011; Deubel et al., 1996). According to the reference object theory (Deubel et al., 1996) or the saccade target theory (Currie, Mcconkie, Carlson-Radvansky \& Irwin, 2000; McConkie \& Currie, 1996), the visual system expects after the saccade the target to be in a local region around the eye landing position (approximatively equivalent to the saccadic endpoint scatter). The assumption that the world remained stable is verified if this prediction is achieved (Wexler \& Collins, 2014). This strategy can operate in the STEP-SAC condition since the saccadic target is directly available at the end of the movement, which may explain why small jumps (that is, within this restricted region) are unnoticeable by the participants (saccadic suppression). In contrast, this strategy is not functional in the case of our GAP-SAC condition performed in a totally dark environment, forcing the visual system to rely more on other (extra-retinal) signals to accurately remap pre-saccadic locations into the post-saccadic reference frame. Thus, the manifestation of the blanking effect in all healthy subjects shows that they can rely on retinal (SSD-STEP task) and extra-retinal (SSD-GAP task) signals in an optimal way to solve these tasks. Moreover, as presented in Introduction, the use of extra-retinal signals is critical to solve the LOC-SAC task as well but, in this case, it is the pre-saccadic location of the probe stimulus (i.e., the bar), not of the saccade target T1 as in the SSD-GAP task, which has to be accurately remapped into the post-saccadic reference frame. In this LOC-SAC task, the performance of $\mathrm{CF}$ within his right visual hemifield was very accurate (figures $7 C$ and 8 ), as revealed by a localization error close to zero. Overall, these results indicate that CF performed the three trans-saccadic localization tasks as accurately as healthy participants when a rightward saccade is performed; this confirms that within his right (ipsi-lesional) visualhemifield, retinal and extra-retinal signals are faithfully integrated to provide accurate perceptual localization judgements. 


\subsection{A deficit of extra-retinal signals for trans-saccadic perceptual judgements within the ataxic visual field}

Thus, based on this framework, if the PPC lesion in patient CF interfered with extratretinal signals, we predicted that: (1) a deficit should occur mostly in the LOC task and in the GAP version of the SSD task where the contribution of extra-retinal signals is supposedly the highest, (2) in the more specific case of the lesion leading to a systematic underestimation of encoding the actual saccade magnitude, the perceptual deficit should be uniform and reflected as a forward bias along the saccade direction, (3) in addition since only the right PPC lesion is symptomatic in $\mathrm{CF}$ and probably leads to his optic ataxia within his left visual hemifield, a perceptual deficit should only occur in his left hemifield. Our results confirmed all these predictions: indeed, CF demonstrated a strong behavioural deficit restricted to his left (contralesional) hemifield in all tasks but predominantly in the GAP and LOC saccadic tasks after subtraction of the corresponding fixation tasks. The localization performance of $\mathrm{CF}$ in the STEP-SAC condition is very accurate (PSS close to zero). The observed difference between CF and healthy individuals under this condition (after subtraction of the corresponding fixation task) could therefore be explained by an alteration of the "phi phenomenon" in the STEP FIX condition -which is absent in the STEP SAC condition due to the saccadic suppression effect- rather than other (extraretinal) factors. Additionally, the analysis of oculomotor behaviour revealed accurate saccades in all tasks (figure 9). Thus, neither a pure visual nor a pure oculomotor factor can account for CF's deficits which, rather, are consistent with a systematic underestimation of the encoding of his actual oculomotor response resulting from erroneous processing and/or integration of extra-retinal signals. 


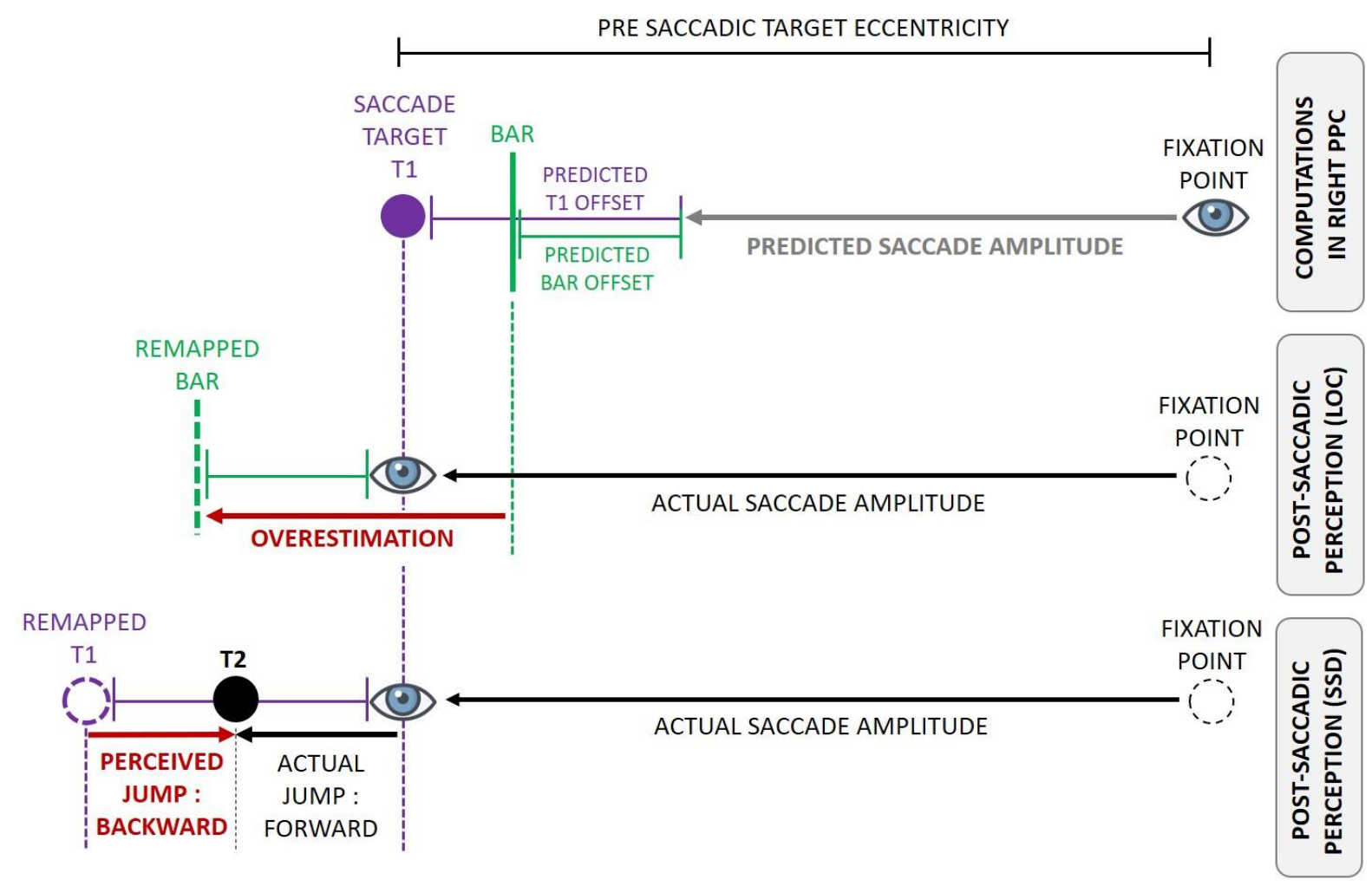

Figure 10. Schematic illustration of CF's altered integration of efference copy for leftward trials of the trans-saccadic tasks. Note that, for the sake of demonstration, the remapping deficit shown here has been isolated from the contribution of initial target location encoding. Specific aspects of the LOC task are shown in green and those of the SSD-GAP task in purple. In the pre-saccadic phase, based on pre saccadic eccentricity encoding (considered here as correct) of stimuli presented during the saccade latency (i.e., the saccade target T1 in SSD-GAP task and the bar in the LOC task) but on an improper corollary discharge (gain $~ 0.67$ according to our data), the expected position of these to-belocalized stimuli relative to the saccade landing position will be wrongly computed by the PPC (shown as 'predicted T1/bar offset'). In the post-saccadic phase, since the saccade is accurately executed, the predicted image will be shifted in the forward direction ('remapped T1/Bar'), leading to strong perceptual biases (red arrows): an overestimation of the position of the bar in the LOC task and an erroneous report in the SSD-GAP task (e.g. as shown here: a backward report for a forward target jump with $T 2$ landing between the actual and the remapped position of T1).

Quite remarkably, CF localization deficits were very similar in both LOC and SSD-GAP tasks in terms of direction (overestimation) and magnitude (constant error of $5.4^{\circ}$ in LOC; PSS of $4.44^{\circ}$ in SSD-GAP). Furthermore, when expressed as a ratio relative to actual saccade size, perceptual error yielded even closer consistent values between the two tasks $(31.6 \%$ in LOC and $33.41 \%$ in SSD-GAP task). This suggests that the lesion induced the same overestimation (mean value: $32.5 \%$ ) of target localization in both tasks, due to an underestimation of the size of the saccade (predicted saccade size representing $67.5 \%$ of the actual size). This underestimation could result either from a reduced gain $(\sim 0.67)$ of extra-retinal signals or from an erroneous integration of those signals. Our interpretation is illustrated schematically in figure 10 for a localization trial in the left (contralesional) hemifield. Note that, since the 
present study allowed us to isolate the sole saccade-related deficit, we do not include the preexistent deficit in fixation condition discussed in Discussion-section 1. We thus assume that in the pre-saccadic phase, patient CF visually encodes both kinds of to-be-localized-stimuli (T1 target and the bar) at their veridical locations. Also, we assume that extra-retinal signals are, at this time, faithfully encoded since the lesion does not involve the corollary discharge- and proprioceptive-related pathways proper. However, due to the lesion, the processing of extraretinal signals by the right PPC is impaired, equivalent to a reduced gain ( $\sim 0.67$ according to our data); this impairment leads to an erroneous prediction of eye saccade amplitude and thus to erroneous predictions of the position of the bar and of the T1 target relative to the saccade landing position (see 'computations in right PPC' on figure 10). After the eye movement is accurately executed, the predicted stimulus position will thus be shifted in the forward direction from the actual saccade landing position ('remapped bar/Tl'). Consequently, in the LOC-task (see 'post-saccadic perception (LOC)'), patient CF will systematically overestimate the position of the bar. Similarly, in the SSD-GAP task (see 'post-saccadic perception $\left.(S S D)^{\prime}\right)$, perceptual reports of target jumps will be biased because the reference position to which the post-saccadic target location (T2) has to compared is the erroneously remapped position of T1. Hence, for example, if $\mathrm{T} 2$ appears between the saccade landing position and the remapped position of $\mathrm{T} 1, \mathrm{CF}$ will perceive a backward jump despite an actual forward target jump. In conclusion, this scheme accounts both qualitatively and quantitatively for the deficits observed in patient $\mathrm{CF}$ in the saccade conditions. It illustrates that the consequences of the parietal lesion on trans-saccadic localization tasks can be condensed as a modification of a single parameter, namely the gain of extra-retinal signals. However, a more comprehensive understanding of the contribution of the parietal cortex should also take into account the (pre-saccadic) localization deficits demonstrated by $\mathrm{CF}$ in the ocular fixation conditions, as discussed in the following section.

\subsection{Parietal involvement in spatial remapping for perceptual judgments}

The present study demonstrates that the human PPC is not only involved in the motor manifestations (such as in the double saccade task) of saccadic remapping processes (see Introduction), but also in their perceptual manifestations. Testing patient $\mathrm{CF}$ both in the ocular fixation and saccadic conditions of our three perceptual tasks disclosed a causal role of the PPC in the processing and integration of retinal and extra-retinal signals for accurate 
perceptual localization performance. This contribution of PPC may be distinct from the frequently suggested deficit of transsaccadic spatial working memory in case of PPC lesion inducing visual neglect (see Pisella \& Mattingley, 2004 and Pisella et al., 2011). Indeed, our patient CF showed no evidence of clinical neglect. In addition, the SSD and LOC tasks used in our study were not designed to isolate specific deficits of spatial working memory. Moreover, if nonetheless present, spatial working memory deficit should be (at least in part) cancelled by the subtraction between saccade and fixation conditions since the temporality of events was similar. Note further that our results showing the gross preservation of saccadic remapping across hemifields in patient CF are consistent with those of Khan et al (2005) in optic ataxia patients. In this 2005 's study, 1) patients had to generate a saccade overpassing the remembered location of a just extinguished target, such that the memory trace of the visual target shifted into the other cerebral hemisphere and then 2) to manually point at this remembered location, a task therefore involving an additional visuo-manual transformation process, known to be impaired in the contralesional field in optic ataxia. When presented in the right (ipsilesional) hemifield, the visual target was accurately encoded and the patient produced a rightward saccade (whose size was also correctly encoded) which overpassed the target. Then the manual reach was performed within the now left (contralesional) hemifield. In this condition, a manual pointing error was observed, resulting solely from the faulty visuomanual transformation of the memorised target location. Now when the visual target was presented in the left (contralesional) hemifield, the patient had to generate a leftward saccade to overpass it and then a manual reach movement toward this target location within the now right (ipsilesional) hemifield. In this condition, the pointing performance was correct (and thus normalised with respect to the immediate pointing to the same left peripheral visual target in fixation condition) because the visual target representation ended in the hemifield where the visuo-manual transformation was preserved. Along this line, Lisi (2020) recently wrote that taken together, the literature shows that « patients with PPC lesions can use extraretinal information to maintain a stable sense of location across eye movements », but nevertheless « do not do so as efficiently as healthy participants, making both more variable and hypometric secondary saccades and more errors in their perceptual judgments ».The present study confirms that saccade remapping mechanisms are preserved in optic ataxia and, through a far more fine-grained analysis disentangling between visual encoding and saccade encoding, further discloses a small but consistent underestimation bias of saccade size encoding. 
Quite remarkably, the deficit of perceptual judgment found in patient CF in the SSD-SAC tasks is very similar to that reported in the same tasks when thalamo-cortical pathways that supposedly carry corollary discharge information are disrupted. Indeed, Ostendorf et al. (2010) tested thalamic patients in SSD tasks and argued that a lesion of the MD nucleus of the thalamus leads to an underestimation of the corollary discharge, by $11 \%$. In another study using TMS in healthy participants, Ostendorf et al. (2011) reported that a disruption of the FEF activity by TMS lead to a similar effect (underestimation by $11.5 \%$ ). In the monkey, transient inactivations of the MD thalamic nucleus yield monkeys to incorrectly predict the perceptual consequences of their saccades in a SSD task (Cavanaugh et al., 2016).

Note finally that in the context of oculomotor performance measured in double saccade paradigms, an alteration of corollary discharge was also suggested in thalamic patients (Bellebaum et al., 2005a, 2006), and the updating of visual space required in those paradigms was proposed, based on EEG recordings in healthy participants, to take place in the PPC (Bellebaum, Hoffmann \& Daum, 2005b). In the context of SSD perceptual performance, whereas Fabius et al. (2020) found no pronounced deficit in a parietal patient group, other studies, using TMS over the PPC, showed evidence of remapping deficit (Chang \& Ro, 2007 ; see also Collins \& Jacquet, 2018).

Altogether, these previous data suggested that thalamo-cortical pathways (such as the MDFEF pathway and a possible thalamo-PPC pathway) carry a corollary discharge underlying trans-saccadic localization performance (perceptual and/or oculomotor). Interestingly, these multiple pathways have been suggested to converge toward the PPC to maintain visual stability across saccades. Ziesche and Hamker (2014) have conceived a neurocomputational model with the parietal cortex as the key node of the visual stability mechanisms. In their model, disabling parietal "remapping cells" (thought to be located in the monkey LIP area according to the neurophysiological literature) either directly or by suppressing their input from extra-retinal streams, should lead to a similar bias than that reported presently in our SSD tasks (i.e., a shift of the psychometric curve in forward direction). Note that the mislocalizations observed in patient $\mathrm{CF}$ were consistent between our tasks and larger (overestimations by $33.53 \%$ in the SSD-GAP task and $31.76 \%$ in the LOC task) than in patients with corollary discharge-related pathway disruption (by about $11 \%$ as reported above). We thus postulate that the right hemispheric lesion in patient CF interfered with these predictive remapping cells for leftward saccades.

Another interesting fact is that patient CF does not experience a breakdown of visual continuity in everyday life, like patients with thalamic lesions (Ostendorf et al., 2010). This 
means that the PPC is probably not the sole or the ultimate structure integrating saccaderelated information to maintain visual stability across saccades. For instance, Berman and Wurtz $(2010,2011)$ identified a functional circuit ascending from superior colliculus to middle temporal area through the pulvinar nucleus that could also sustain visual stability. Other mechanisms than alternative corollary discharge pathways, such as relative localization to visual landmarks in real scenes (Bays \& Husain, 2007) or sustained visual attention (Rolfs, Jonikaitis, Deubel \& Cavanagh, 2010) may contribute to perceptual visual stability. Altogether, this could probably explain why only large and bilateral brain damage affects the subjective impression of visual constancy across saccades (Haarmeier, Thier, Repnow \& Petersen, 1997), particularly in conditions with no visual landmarks, like in the present study, forcing the CNS to use and integrate the corollary discharge with the remembered location of a stimulus.

To conclude, this study extents previous works and offers direct experimental supports for an important contribution of the PPC in trans-saccadic perceptual judgments of visual space by integrating several visual and extra-retinal signals.

\section{Credit Author Statement}

Alexis Cheviet: Conceptualization, Methodology, Software, Development, Formal analysis, Investigation, Writing - Original Draft. Laure Pisella: Conceptualization, Methodology, Resources, Writing - Original Draft, Supervision. Denis Pélisson: Conceptualization, Methodology, Resources, Writing - Original Draft, Supervision.

\section{Acknowledgements}

The authors thank Eric Koun and Roméo Salemme for their assistance with the practical implementation of the protocol and their advice about tools to use for eye-movements analysis. This work was supported by the Lyon Neuroscience Research Center, INSERM u1028, CNRS -UMR 5292, University Claude Bernard Lyon 1, 69 676, France. Author A.C received funding from the 'Agence Nationale de la Recherche' grant to the author DP (ANR15-CE37-0014-01) and from Fondation de France - Berthe Fouassier scholarship (2019, 00099566). Declarations of interest: none. No part of the study procedures and analyses was pre-registered prior to the research being conducted. 


\section{REFERENCES}

1. Awater, H. \& Lappe, M. (2006). Mislocalization of Perceived Saccade Target Position Induced by Perisaccadic Visual Stimulation. Journal of Neuroscience, 26(1), 12-20. doi: 10.1523/jneurosci.2407-05.2006

2. Battelli, L., Cavanagh, P., Intriligator, J., Tramo, M. J., Hénaff, M.-A., Michèl, F., \& Barton, J. J. (2001). Unilateral Right Parietal Damage Leads to Bilateral Deficit for High-Level Motion. Neuron, 32(6), 985-995. doi: 10.1016/s0896-6273(01)00536-0

3. Bays, P. M., \& Husain, M. (2007). Spatial remapping of the visual world across saccades. NeuroReport, 18(12), 1207-1213. doi: 10.1097/wnr.0b013e328244e6c3

4. Becker, W., \& Jürgens, R. (1979). An analysis of the saccadic system by means of double step stimuli. Vision Research, 19(9), 967-983. doi: 10.1016/00426989(79)90222-0

5. Bellebaum, C., Daum, I., Koch, B., Schwarz, M., \& Hoffmann, K.-P. (2005a). The role of the human thalamus in processing corollary discharge. Brain, 128(5), 11391154. doi: 10.1093/brain/awh474

6. Bellebaum, C., Hoffmann, K.-P., \& Daum, I. (2005b). Post-saccadic updating of visual space in the posterior parietal cortex in humans. Behavioural Brain Research, 163(2), 194-203. doi: 10.1016/j.bbr.2005.05.007

7. Bellebaum, C., Hoffmann, K.-P., Koch, B., Schwarz, M., \& Daum, I. (2006). Altered processing of corollary discharge in thalamic lesion patients. European Journal of Neuroscience, 24(8), 2375-2388. doi: 10.1111/j.1460-9568.2006.05114.x

8. Berman, R. A., \& Wurtz, R. H. (2010). Functional Identification of a Pulvinar Path from Superior Colliculus to Cortical Area MT. Journal of Neuroscience, 30(18), 6342-6354. doi: 10.1523/jneurosci.6176-09.2010

9. Berman, R. A., \& Wurtz, R. H. (2011). Signals Conveyed in the Pulvinar Pathway from Superior Colliculus to Cortical Area MT. Journal of Neuroscience, 31(2), 373384. doi: 10.1523/jneurosci.4738-10.2011

10. Blangero, A., Ota, H., Rossetti, Y., Fujii, T., Ohtake, H., Tabuchi, M., ... Pisella, L. (2010). Systematic retinotopic reaching error vectors in unilateral optic ataxia. Cortex, 46(1), 77-93. doi: 10.1016/j.cortex.2009.02.015

11. Blangero, A., Gaveau, V., Luauté, J., Rode, G., Salemme, R., Guinard, M., ... Pisella, L. (2008). A hand and a field effect in on-line motor control in unilateral optic ataxia. Cortex, 44(5), 560-568. doi: 10.1016/j.cortex.2007.09.004 
12. Cavanaugh, J., Berman, R. A., Joiner, W. M., \& Wurtz, R. H. (2016). Saccadic Corollary Discharge Underlies Stable Visual Perception. Journal of Neuroscience, 36(1), 31-42. doi: 10.1523/jneurosci.2054-15.2016

13. Chang, E., \& Ro, T. (2007). Maintenance of Visual Stability in the Human Posterior Parietal Cortex. Journal of Cognitive Neuroscience, 19(2), 266-274. doi: 10.1162/jocn.2007.19.2.266

14. Churan, J., Guitton, D., \& Pack, C. C. (2012). Perisaccadic Remapping and Rescaling of Visual Responses in Macaque Superior Colliculus. PLoS ONE, 7(12). doi: 10.1371/journal.pone.0052195

15. Collins, T., \& Jacquet, P. O. (2018). TMS over posterior parietal cortex disrupts transsaccadic visual stability. Brain Stimulation, 11(2), 390-399. doi: 10.1016/j.brs.2017.11.019

16. Collins, T., Rolfs, M., Deubel, H., \& Cavanagh, P. (2009). Post-saccadic location judgments reveal remapping of saccade targets to non-foveal locations. Journal of Vision, 9(5), 29-29. doi: 10.1167/9.5.29

17. Crawford, J., \& Garthwaite, P. H. (2002). Investigation of the single case in neuropsychology: confidence limits on the abnormality of test scores and test score differences. Neuropsychologia, 40(8), 1196-1208. doi: 10.1016/s00283932(01)00224-X

18. Currie, C. B., Mcconkie, G. W., Carlson-Radvansky, L. A., \& Irwin, D. E. (2000). The role of the saccade target object in the perception of a visually stable world. Perception \& Psychophysics, 62(4), 673-683. doi: 10.3758/bf03206914

19. Deubel, H., Schneider, W. X., \& Bridgeman, B. (1996). Postsaccadic target blanking prevents saccadic suppression of image displacement. Vision Research, 36(7), 985996. doi: 10.1016/0042-6989(95)00203-0

20. Deubel, H., Schneider, W. X., \& Bridgeman, B. (2002). Transsaccadic memory of position and form. The Brains Eye: Neurobiological and Clinical Aspects of Oculomotor Research Progress in Brain Research, 165-180. doi: 10.1016/s00796123(02)40049-0

21. Dijkerman, H., Mcintosh, R., Anema, H., Haan, E. D., Kappelle, L., \& Milner, A. (2006). Reaching errors in optic ataxia are linked to eye position rather than head or body position. Neuropsychologia, 44(13), 2766-2773. doi: 10.1016/j.neuropsychologia.2005.10.018 
22. Duhamel, J., Colby, C., \& Goldberg, M. (1992a). The updating of the representation of visual space in parietal cortex by intended eye movements. Science, 255(5040), 9092. doi: $10.1126 /$ science. 1553535

23. Duhamel, J., Goldberg, M. E., Fitzgibbon, E. J., Sirigu, A., \& Grafman, J. (1992b). Saccadic Dysmetria In A Patient With A Right Frontoparietal Lesion. Brain, 115(5), 1387-1402. doi: 10.1093/brain/115.5.1387

24. Fabius, J. H., Nijboer, T. C. W., Fracasso, A., \& Van der Stigchel, S. (2020). Intrasaccadic displacement sensitivity after a lesion to the posterior parietal cortex. Cortex, 127, 108-119. doi: 10.1016/j.cortex.2020.01.027

25. Gaveau, V., Pélisson, D., Blangero, A., Urquizar, C., Prablanc, C., Vighetto, A., \& Pisella, L. (2008). Saccade control and eye-hand coordination in optic ataxia. Neuropsychologia, 46(2), 475-486. doi: 10.1016/j.neuropsychologia.2007.08.028

26. Gaymard, B., Rivaud, S., \& Pierrot-Deseilligny, C. (1994). Impairment of extra-retinal eye position signals after central thalamic lesions in humans. Experimental Brain Research, 102(1). doi: 10.1007/bf00232433

27. Goldberg, M. E., \& Bruce, C. J. (1990). Primate frontal eye fields. III. Maintenance of a spatially accurate saccade signal. Journal of Neurophysiology, 64(2), 489-508. doi: 10.1152/jn.1990.64.2.489

28. Granek, J. A., Pisella, L., Blangero, A., Rossetti, Y., \& Sergio, L. E. (2012). The Role of the Caudal Superior Parietal Lobule in Updating Hand Location in Peripheral Vision: Further Evidence from Optic Ataxia. PLoS ONE, 7(10). doi: 10.1371/journal.pone.0046619

29. Haarmeier, T., Thier, P., Repnow, M., \& Petersen, D. (1997). False perception of motion in a patient who cannot compensate for eye movements. Nature, 389(6653), 849-852. doi: 10.1038/39872

30. Hallett, P., \& Lightstone, A. (1976). Saccadic eye movements to flashed targets. Vision Research, 16(1), 107-114. doi: 10.1016/0042-6989(76)90084-5

31. Hamker, F. H., Zirnsak, M., Ziesche, A., \& Lappe, M. (2011). Computational models of spatial updating in peri-saccadic perception. Philosophical Transactions of the Royal Society B: Biological Sciences, 366(1564), 554-571. doi: 10.1098/rstb.2010.0229

32. Heide, W., Blankenburg, M., Zimmermann, E., \& Kömpf, D. (1995). Cortical control of double-step saccades: Implications for spatial orientation. Annals of Neurology, 38(5), 739-748. doi: 10.1002/ana.410380508 
33. Honda, H. (1991). The time courses of visual mislocalization and of extra-retinal eye position signals at the time of vertical saccades. Vision Research, 31(11), 1915-1921. doi: 10.1016/0042-6989(91)90186-9

34. Khan, A. Z., Pisella, L., Vighetto, A., Cotton, F., Luauté, J., Boisson, D., .. Rossetti, Y. (2005a). Optic ataxia errors depend on remapped, not viewed, target location. Nature Neuroscience, 8(4), 418-420. doi: 10.1038/nn1425

35. Khan, A., Blangero, A., Rossetti, Y., Salemme, R., Luaute, J., Deubel, H., ... Pisella, L. (2008). Parietal Damage Dissociates Saccade Planning from Presaccadic Perceptual Facilitation. Cerebral Cortex, 19(2), 383-387. doi: 10.1093/cercor/bhn088

36. Khan, A. Z., Pisella, L., Rossetti, Y., Vighetto, A., \& Crawford, J. D. (2005b). Impairment of Gaze-centered Updating of Reach Targets in Bilateral ParietalOccipital Damaged Patients. Cerebral Cortex, 15(10), 1547-1560. doi: 10.1093/cercor/bhi033

37. Khan, A. Z., Pisella, L., \& Blohm, G. (2013). Causal evidence for posterior parietal cortex involvement in visual-to-motor transformations of reach targets. Cortex, 49(9), 2439-2448. doi: 10.1016/j.cortex.2012.12.004

38. Knapen, T., Swisher, J. D., Tong, F., \& Cavanagh, P. (2016). Oculomotor Remapping of Visual Information to Foveal Retinotopic Cortex. Frontiers in Systems Neuroscience, 10. doi: 10.3389/fnsys.2016.00054

39. Lisi, M. (2020). Uncertainty and spatial updating in posterior parietal cortex. Cortex, 130, 441- 443. https://doi.org/10.1016/j.cortex.2020.02.013

40. Mays, L. E., \& Sparks, D. L. (1980). Dissociation of visual and saccade-related responses in superior colliculus neurons. Journal of Neurophysiology, 43(1), 207-232. doi: 10.1152/jn.1980.43.1.207

41. Mcconkie, G. W., \& Currie, C. B. (1996). Visual stability across saccades while viewing complex pictures. Journal of Experimental Psychology: Human Perception and Performance, 22(3), 563-581. doi: 10.1037/0096-1523.22.3.563

42. Medendorp, W. P., Goltz, H. C., Vilis, T., \& Crawford, J. D. (2003). Gaze-Centered Updating of Visual Space in Human Parietal Cortex. The Journal of Neuroscience, 23(15), 6209-6214. doi: 10.1523/jneurosci.23-15-06209.2003

43. Merriam, E. P., Genovese, C. R., \& Colby, C. L. (2003). Spatial Updating in Human Parietal Cortex. Neuron, 39(2), 361-373. doi: 10.1016/s0896-6273(03)00393-3 
44. Michel, F., \& Henaff, M.-A. (2004). Seeing without the Occipito-Parietal Cortex: Simultagnosia as a Shrinkage of the Attentional Visual Field. Behavioural Neurology, 15(1-2), 3-13. doi: 10.1155/2004/836830

45. Milner, A. D., Paulignan, Y., Dijkerman, H. C., Michel, F., \& Jeannerod, M. (1999). A paradoxical improvement of misreaching in optic ataxia: new evidence for two separate neural systems for visual localization. Proceedings of the Royal Society of London. Series B: Biological Sciences, 266(1434), 2225-2229. doi: 10.1098/rspb.1999.0912

46. Milner, A., Dijkerman, H., Mcintosh, R., Rossetti, Y., \& Pisella, L. (2003). Delayed reaching and grasping in patients with optic ataxia. Progress in Brain Research Neural Control of Space Coding and Action Production, 225-242. doi: 10.1016/s00796123(03)42016-5

47. Morris, A. P., Chambers, C. D., \& Mattingley, J. B. (2007). Parietal stimulation destabilizes spatial updating across saccadic eye movements. Proceedings of the National Academy of Sciences, 104(21), 9069-9074. doi: 10.1073/pnas.0610508104

48. Morrone, M. C., Ross, J., \& Burr, D. C. (1997). Apparent Position of Visual Targets during Real and Simulated Saccadic Eye Movements. The Journal of Neuroscience, 17(20), 7941-7953. doi: 10.1523/jneurosci.17-20-07941.1997

49. Nakamura, K., \& Colby, C. L. (2002). Updating of the visual representation in monkey striate and extrastriate cortex during saccades. Proceedings of the National Academy of Sciences, 99(6), 4026-4031. doi: 10.1073/pnas.052379899

50. Ostendorf, F., Liebermann, D., \& Ploner, C. J. (2010). Human thalamus contributes to perceptual stability across eye movements. Proceedings of the National Academy of Sciences, 107(3), 1229-1234. doi: 10.1073/pnas.0910742107

51. Ostendorf, F., Kilias, J., \& Ploner, C. J. (2011). Theta-Burst Stimulation over Human Frontal Cortex Distorts Perceptual Stability across Eye Movements. Cerebral Cortex, 22(4), 800-810. doi: 10.1093/cercor/bhr143

52. Ostendorf, F., Liebermann, D., \& Ploner, C. J. (2013). A role of the human thalamus in predicting the perceptual consequences of eye movements. Frontiers in Systems Neuroscience, 7. doi: 10.3389/fnsys.2013.00010

53. Pierce, J. E., Saj, A., \& Vuilleumier, P. (2019). Differential parietal activations for spatial remapping and saccadic control in a visual memory task. Neuropsychologia, 131, 129-138. doi: 10.1016/j.neuropsychologia.2019.05.010 
54. Pisella, L., Alahyane, N., Blangero, A., Thery, F., Blanc, S., \& Pelisson, D. (2011). Right-hemispheric dominance for visual remapping in humans. Philosophical Transactions of the Royal Society B: Biological Sciences, 366(1564), 572-585. doi: 10.1098/rstb.2010.0258

55. Pisella, L., \& Mattingley, J. B. (2004). The contribution of spatial remapping impairments to unilateral visual neglect. Neuroscience \& Biobehavioral Reviews, 28(2), 181-200. doi: 10.1016/j.neubiorev.2004.03.003

56. Podvalny, E., Yeagle, E., Mégevand, P., Sarid, N., Harel, M., Chechik, G., ... Malach, R. (2017). Invariant Temporal Dynamics Underlie Perceptual Stability in Human Visual Cortex. Current Biology, 27(2), 155-165. doi: 10.1016/j.cub.2016.11.024

57. Prime, S. L., Vesia, M., \& Crawford, J. D. (2008). Transcranial Magnetic Stimulation over Posterior Parietal Cortex Disrupts Transsaccadic Memory of Multiple Objects. Journal of Neuroscience, 28(27), 6938-6949. doi: 10.1523/jneurosci.0542-08.2008

58. Rao, H. M., Mayo, J. P., \& Sommer, M. A. (2016). Circuits for presaccadic visual remapping. Journal of Neurophysiology, 116(6), 2624-2636. doi: 10.1152/jn.00182.2016

59. Rath-Wilson, K., \& Guitton, D. (2015a). Refuting the hypothesis that a unilateral human parietal lesion abolishes saccade corollary discharge. Brain, 138(12), 37603775. doi: 10.1093/brain/awv275

60. Rath-Wilson, K., \& Guitton, D. (2015b). Oculomotor control after hemidecortication: A single hemisphere encodes corollary discharges for bilateral saccades. Cortex, 63, 232-249. doi: 10.1016/j.cortex.2014.08.020

61. Rolfs, M., Jonikaitis, D., Deubel, H., \& Cavanagh, P. (2010). Predictive remapping of attention across eye movements. Nature Neuroscience, 14(2), 252-256. doi: $10.1038 / \mathrm{nn} .2711$

62. Rossetti, Y., Revol, P., Mcintosh, R., Pisella, L., Rode, G., Danckert, J., ... Milner, A. (2005). Visually guided reaching: bilateral posterior parietal lesions cause a switch from fast visuomotor to slow cognitive control. Neuropsychologia, 43(2), 162-177. doi: 10.1016/j.neuropsychologia.2004.11.004

63. Sommer, M. A. (2002). A Pathway in Primate Brain for Internal Monitoring of Movements. Science, 296(5572), 1480-1482. doi: 10.1126/science.1069590

64. Sommer, M. A., \& Wurtz, R. H. (2008). Brain Circuits for the Internal Monitoring of Movements. Annual Review of Neuroscience, 31(1), 317-338. doi:

10.1146/annurev.neuro.31.060407.125627 
65. Sperry, R. W. (1950). Neural basis of the spontaneous optokinetic response produced by visual inversion. Journal of Comparative and Physiological Psychology, 43(6), 482-489. doi: 10.1037/h0055479

66. Ten Brink, A. F., Fabius, J. H., Weaver, N. A., Nijboer, T. C. W., \& Van der Stigchel, S. (2019). Trans-saccadic memory after right parietal brain damage. Cortex, 120, 284-297. doi: 10.1016/j.cortex.2019.06.006

67. Tolias, A. S., Moore, T., Smirnakis, S. M., Tehovnik, E. J., Siapas, A. G., \& Schiller, P. H. (2001). Eye Movements Modulate Visual Receptive Fields of V4 Neurons. Neuron, 29(3), 757-767. doi: 10.1016/s0896-6273(01)00250-1

68. Umeno, M. M., \& Goldberg, M. E. (1997). Spatial Processing in the Monkey Frontal Eye Field. I. Predictive Visual Responses. Journal of Neurophysiology, 78(3), 13731383. doi: 10.1152/jn.1997.78.3.1373

69. Van Donkelaar, P., \& Müri, R. (2002). Craniotopic updating of visual space across saccades in the human posterior parietal cortex. Proceedings of the Royal Society of London. Series B: Biological Sciences, 269(1492), 735-739. doi: 10.1098/rspb.2001.1935

70. Vindras, P., Blangero, A., Ota, H., Reilly, K. T., Rossetti, Y., \& Pisella, L. (2016). The Pointing Errors in Optic Ataxia Reveal the Role of "Peripheral Magnification" of the PPC. Frontiers in Integrative Neuroscience, 10. doi: 10.3389/fnint.2016.00027

71. Von Holst, E., \& Mittelstaedt, H. (1950). Das Reafferenzprinzip. Naturwissenschaften, 37(20), 464-476. doi: 10.1007/bf00622503

72. Walker, M. F., Fitzgibbon, E. J., \& Goldberg, M. E. (1995). Neurons in the monkey superior colliculus predict the visual result of impending saccadic eye movements. Journal of Neurophysiology, 73(5), 1988-2003. doi: 10.1152/jn.1995.73.5.1988

73. Wexler, M., \& Collins, T. (2014). Orthogonal steps relieve saccadic suppression. Journal of Vision, 14(2), 13-13. doi: 10.1167/14.2.13

74. Wichmann, F. A., \& Hill, N. J. (2001). The psychometric function: I. Fitting, sampling, and goodness of fit. Perception \& Psychophysics, 63(8), 1293-1313. doi: 10.3758/bf03194544

75. Wurtz, R. H. (2008). Neuronal mechanisms of visual stability. Vision Research, 48(20), 2070-2089. doi: 10.1016/j.visres.2008.03.021

76. Ziesche, A., \& Hamker, F. H. (2011). A Computational Model for the Influence of Corollary Discharge and Proprioception on the Perisaccadic Mislocalization of Briefly 
Presented Stimuli in Complete Darkness. Journal of Neuroscience, 31(48), 1739217405. doi: 10.1523/jneurosci.3407-11.2011

77. Ziesche, A., \& Hamker, F. H. (2014). Brain circuits underlying visual stability across eye movements: converging evidence for a neuro-computational model of area LIP. Frontiers in Computational Neuroscience, 8. doi: 10.3389/fncom.2014.00025

78. Ziesche, A., Bergelt, J., Deubel, H., \& Hamker, F. H. (2017). Pre- and post-saccadic stimulus timing in saccadic suppression of displacement - A computational model. Vision Research, 138, 1-11. doi: 10.1016/j.visres.2017.06.007

79. Zimmerman, E., \& Lappe, M. (2010). Motor signals in visual localization. Journal of Vision, 10(6), 2-2. doi: 10.1167/10.6.2

80. Zimmermann, E., Weidner, R., Abdollahi, R. O., \& Fink, G. R. (2016). Spatiotopic Adaptation in Visual Areas. Journal of Neuroscience, 36(37), 9526-9534. doi: 10.1523/jneurosci.0052-16.2016 